\title{
About frame estimation of growth functions and robust prediction in bioprocess modeling
}

\author{
Krichen Emna ${ }^{1,2}$, Rapaport A. ${ }^{3,}{ }^{*}$, Fouilland Eric ${ }^{2}$
}

1 Univ Montpellier, INRA, SupAgro, MISTEA, Montpellier, France.

2 Univ Montpellier, IFREMER, CNRS, IRD,MARBEC, Montpellier, France.

${ }^{3}$ Univ Montpellier, INRA, SupAgro, MISTEA, Montpellier, France.

*Corresponding author : A. Rapaport, email address : alain.rapaport@inra.fr emna.krichen@supagro.fr ; eric.fouilland@cnrs.fr

\begin{abstract}
:
We address the problem of determining functional framing from experimental data points in view of robust time-varying predictions, which is of crucial importance in bioprocess monitoring. We propose a method that provides guaranteed functional bounds, instead of sets of parameters values for growth functions such as the classical Monod or Haldane functions commonly used in bioprocess modeling. We illustrate the applicability of the method with bioreactor simulations in batch and continuous mode, as well as on real data. We also present two extensions of the method adding flexibility in its application, and discuss its efficiency in providing guaranteed state estimations.
\end{abstract}

\section{Highlights}

- We propose a method to obtain functional frames of growth functions from experimental data. Data points are wrapped by graphs of 2 functions without requiring any assumption on the unkwown growth function. Some additional flexibilities of the method are introduced. The approach is illustrated on synthetic and real data for robust predictions.

Keywords : Functional estimation, Interval observers, Growth functions, Least square 


\section{Introduction and motivations}

Interval analysis has recently gained a lot of popularity for the robust estimation of state variables in dynamical models. Bioprocesses are typically subject to various kind of variations or disturbances which are not always easy to model $[3,9]$. The idea to determine or compute bounds for uncertain systems is not new $[40,25,52]$, but a recent renewed focus has been brought to estimate with intervals state variables or parameters from real-time observations, despite uncertainties on the dynamics $[17,18,43,22,49]$. In situations for which the usual random representations (based on probability measures, Gaussian processes, white noise...) are not appropriate or cannot be justified for modeling the perturbations, interval observers give a simple and attractive alternative [13]. Instead of (non-robust) single-valued observers or stochastic filters which both provide single estimates expected to represent average values (provided that hypotheses on the randomness are satisfied), interval observers give a set of plausible trajectories and/or parameters $[13,30,35,8,55]$. Typically, for a given deterministic model

$$
\frac{d X}{d t}=F(t, X), \quad X \in \mathbb{R}^{n},
$$


where some terms of the map $F$ are badly known or subject to fluctuations, and an observation vector

$$
Y(t)=G(t, X(t)) \in \mathbb{R}^{p}
$$

that is assumed to be available at any time $t$, the interval approach consists in designing two estimators, a "lower" and a "upper" ones, of the form

$$
\frac{d X^{-}}{d t}=F^{-}\left(t, X^{-}, Y(t)\right), \quad \frac{d X^{+}}{d t}=F^{+}\left(t, X^{+}, Y(t)\right)
$$

when the dynamics $F$ is monotone [54, 24], or in a coupled form

$$
\frac{d X^{-}}{d t}=F^{-}\left(t, X^{-}, X^{+}, Y(t)\right), \quad \frac{d X^{+}}{d t}=F^{+}\left(t, X^{-}, X^{+}, Y(t)\right)
$$

when $F$ is non monotone (applying Muller's Theorem, see [40, 56, 30]). In this formulation, $X^{-}, X^{+}$are vectors in $\mathbb{R}^{n}$ which provide a guaranteed framing of the solutions $X(\cdot)$ of $(1)$ in the following sense

$$
\begin{aligned}
& \left\{X_{i}^{-}(0) \leq X_{i}(0) \leq X_{i}^{+}(0),(i=1 \cdots n)\right\} \\
& \quad \Longrightarrow\left\{X_{i}^{-}(t) \leq X_{i}(t) \leq X_{i}^{+}(t),(i=1 \cdots n)\right\}, \quad t>0
\end{aligned}
$$

This approach is well suited to "slow" processes or dynamics with long transients, such as bioprocesses, because it guarantees bounds during the transients. It has been successfully applied to several models of bioprocesses $[13,5,15,45$, $33,44,29,10,26,6]$. A possible drawback of this approach is that it could provide quite conservative bounds that could be then uninformative for practitioners. However, in recent years, much progress has been made to improve the width of the guaranteed intervals, playing with different structures of the systems [42, 28, 14, 58] (there exist also several results for the class of linear dynamics), changes of coordinates [47, 8], considerations of bundles of observers $[4,34]$ or with the help of purely numerical methods [21, 22, 43, 44, 30] based on interval analysis $[37,18]$.

In mass balance models for bioprocesses [3, 7], the most critical uncertainty is primarily found on the specific growth functions of the micro-organisms, often denoted by $\mu(\cdot)$. Consider for instance the classical chemostat model $[16]$

$$
\frac{d X}{d t}=F(t, X):=\left[\begin{array}{c}
\mu(t, X) X_{1}-D(t) X_{1} \\
-\frac{1}{r} \mu(t, X) X_{1}+D(t)\left(X_{2}^{i n}-X_{2}\right)
\end{array}\right]
$$

where $X_{1}, X_{2}$ denote the concentrations of biomass and substrate, respectively. The dilution rate $D(\cdot)$, the input substrate concentration $X_{2}^{i n}$ and the yield factor $r$, are supposed to be known. Several contributions among the ones cited previously have considered that the effective growth functions $\mu(\cdot)$ depends on time and on the substrate concentration $s=X_{2}$ in an unknown manner, but are bounded by two "extreme" functions $\mu^{-}(\cdot), \mu^{+}(\cdot)$ so that the inequalities

$$
\mu^{-}(s) \leq \mu(t, s) \leq \mu^{+}(s), \quad s \geq 0
$$

are fulfilled at time $t$, whatever could be the values of the variable $s$. Robust state estimations can then be derived from the knowledge of $\mu^{-}(\cdot)$ and $\mu^{+}(\cdot)$ 
(instead of $\mu(\cdot)$ ), designing the maps $F^{-}, F^{+}$in (3)-(4), as illustrated in [46, $1,45]$. However, one may wonder how to obtain these functional bounds from experimental data. Let us underline that it is particularly relevant to make a good choice of the functions $\mu^{-}, \mu^{+}$as it impacts on the width of the frame (5). Take as an example the class of Monod growth functions

$$
\mu(s)=\frac{\mu_{\max } s}{K_{s}+s}
$$

for which uncertainty is typically on the parameters $\mu_{\max }$ and $K_{s}$. In the context of state or parameter estimation, several studies have focused on developing techniques to improve the computational efficiency when fitting models to measurement data, assuming arbitrary but bounded error distribution $[57,38,19,32,41,27]$. Central of these approaches is overcoming convergence to local optima and inferring joint confidence intervals or regions [57, 12, 41]. It consists then in obtaining either parameter intervals $\left[\mu_{\max }^{-}, \mu_{\max }^{+}\right],\left[K_{s}^{-}, K_{s}^{+}\right]$ in $\mathbb{R}_{+}$or a region of $\mathbb{R}_{+} \times \mathbb{R}_{+}$.

Differently from these techniques of guaranteed parameters estimations, the approach we propose here revolves around a functional framing. In fact, it is no longer a matter of looking for a set of parameters, but rather ensuring that there would exist functions $\mu(\cdot)$ (depending on time in an unknown way) that guarantee that experimental data can be generated by a functional frame. This amounts to look for functional intervals $\left[\mu^{-}(\cdot), \mu^{+}(\cdot)\right]$ within a given class of functions. Although we shall consider classes of functions described by parameters, such as $\mu_{\max }$ and $K_{s}$ for the Monod functions, we shall simply look for two functions $\mu^{-}(\cdot), \mu^{+}(\cdot)$ belonging to this class. Each of them will be characterized by a pair of parameters $\left(\mu_{\max }^{-}, K_{s}^{-}\right),\left(\mu_{\max }^{+}, K_{s}^{+}\right)$, but this does not mean that we shall consider that the unknown $\mu(\cdot)$ is a Monod function with unkwnown parameters $\left(\mu_{\max }, K_{s}\right)$ belonging to a particular subset. We rather state that the unknown growth function is any function $\mu(\cdot)$ verifying the condition (7). In particular $\mu(\cdot)$ is not necessarily a Monod function. Moreover, let us underline that one does not have to impose $\mu_{\max }^{+}>\mu_{\max }^{-}, K_{s}^{+}>K_{s}^{-}$ to obtain inequalities (7) for a given interval of plausible values of $s$ (see also Remark 1 below). To our knowledge, the question of determining functional intervals from experimental data in this way has not been yet addressed in the literature. Functional bounds rather than a parameter set appears to be well suited to the method of interval estimators in the context of bioprocesses.

The purpose of the present work is to propose a generic method to fit two models (an "upper" and a "lower" one) that wrap data within lower and upper functions $\mu^{-}(\cdot)$ and $\mu^{+}(\cdot)$ instead of a single average $\mu(\cdot)$. To avoid too conservative bounds, we also introduce some flexibility in the application of the method for choosing the framing quality, either considering different upper and lower classes of growth functions, or forcing to have tightened bounds across the data sets, or both. We illustrate our framing method on synthetic data generated by random Monod [36] or Haldane [2] growth functions, this latter one including the effect of substrate inhibition. Then, we compute time-varying predictions through numerical simulations of batch and continuous operating mode, providing bounded state estimations. As recalled previously, we mainly target situations for which the usual statistical hypotheses (reproducibility, uniform distribution of samples, unbiased noise...) are not necessarily met to justify the 
usual identification methods (least-squares, coefficient of determination, confidence intervals, maximum likelihood... see for instance $[11,53])$. The approach we propose is purely geometrical and does not rely on any statistical property, even though there are some similitude with least-square methods in the choice of the fitting criterion.

The paper is organized as follows. In Section 2, we present the method in a general framework and its theoretical justification (Propositions 1 and 2). In Section 3, we provide two extensions of the method. Then, Section 4 is dedicated to the application of the method to the framing of growth curves and its use for robust state predictions. Finally, we illustrate and discuss the approach using numerical data and simulations for two types of growth processes (batch and continuous modes) in Section 5 with an application on real data in Section 6 . We end by a conclusion and some perspectives.

\section{Presentation of the method}

We describe here the method in a general setting of an unknown scalar function $f: x \mapsto y=f(x)$, to be bounded by two functions $f^{-}, f^{+}$, given a set of $n$ experimental measurements

$$
\mathcal{S}:=\left\{\left(x_{i}, y_{i}\right)\right\}_{i \in\{1, \cdots, n\}} .
$$

We consider a class $\mathcal{C}$ of functions (also denoted by $f$ for convenience) parameterized by a vector of parameters $p \in P$, where $P$ is a subset of $\mathbb{R}^{m}$ :

$$
\mathcal{C}:=\bigcup_{p \in P}\{f(\cdot, p): \mathbb{R} \mapsto \mathbb{R}\}
$$

and require the following regularity conditions.

Assumption 1. The set $P$ is a compact subset of $\mathbb{R}^{m}$. The map $f$ is continuous with respect to $(x, p)$ in $\mathbb{R} \times P$.

Most of the time, the set $P$ of parameters is simply a cartesian product of intervals.

A frame of $\mathcal{S}$ by functions in $\mathcal{C}$ is determined by a pair $\left(p^{-}, p^{+}\right) \in P^{2}$ that satisfies the constraints

$$
f\left(x_{i}, p^{-}\right) \leq y_{i} \leq f\left(x_{i}, p^{+}\right), \quad i=1 \cdots n .
$$

Remark 1. In bounded error estimation problems (see for instance [5\%, 38]), one usually looks for a subset $\hat{P} \subset P$ of parameters such that one has

$$
\left|f\left(x_{i}, p\right)-y_{i}\right| \leq e, \quad i=1 \cdots n, \quad p \in \hat{P}
$$

where $e>0$ is some tolerance. Then, for a sufficiently large e, the set

$$
\{(x, f(x, p)) ; p \in \hat{P}, x \in \mathbb{R}\}
$$

may contain all the data points $\left(x_{i}, y_{i}\right)$. Notice that the subset $\hat{P}$ has no reason to be a product of intervals. Take for instance the Monod functions (8) and 
assume that data points are contained exactly between the graphs of $\mu^{-}(s)=\frac{s}{1+s}$ and $\mu^{+}(s)=\frac{2 s}{2+s}$. Then, the set of parameters $\left(\mu_{\max }, K_{s}\right)$ such that one has $\mu^{-}(s) \leq \mu(s) \leq \mu^{+}(s)$ for any $s>0$ is not of the form $\left[\mu_{\max }^{-}, \mu_{\max }^{+}\right] \times\left[K_{s}^{-}, K_{s}^{+}\right]$ but is a line segment: $\left\{\left(\mu_{\max }, K_{s}\right) ; \mu_{\max } \in[1,2], K_{s}=\mu_{\max }\right\}$. However, the determination of $\hat{P}$ can be quite difficult when the monotony of $f$ with respect to a parameter changes with $x$ : consider for instance the class of Hill or Moser functions as an extension of the Monod functions [39]:

$$
\mu(s)=\frac{\mu_{\max } s^{\alpha}}{K_{s}+s^{\alpha}}, \quad \mu_{\max }>0, \quad \alpha \geq 1
$$

and data exactly framed by $\mu^{-}(s)=\frac{s^{2}}{1+s^{2}}$ and $\mu^{+}(s)=\frac{2 s}{1+s}$. Here, we do not look for a subset $\hat{P}$, but only to two functions $f^{-}, f^{+}$in the set $\mathcal{C}$ such that the frame (10) is fulfilled.

Our objective is to propose a fast and reliable method to obtain tight frames, taking into consideration that $n$ could be large. Typically, one may face data from experiments conducted in different conditions, each of them with triplicates or more. This could easily lead to hundreds of measurements, as it is the case with microwell plate batch cultures which are quite popular in microbiology. However, we do not impose any restriction of considering small $n$. One may look for the tightest frames, considering a measure of the frames as

$$
D\left(p^{-}, p^{+}\right):=\sum_{i=1}^{n}\left(f\left(x_{i}, p^{+}\right)-f\left(x_{i}, p^{-}\right)\right)^{2}
$$

and the optimization problem

$$
\min \left\{D\left(p^{-}, p^{+}\right) ;\left(p^{-}, p^{+}\right) \in P^{2} \text { satisfying }(10)\right\} .
$$

This is a non-linear optimization problem with $2 n$ non-linear constraints. Such problems are classically addressed considering $2 n$ Karush-Kuhn-Tucker multipliers $\lambda_{i}^{-} \geq 0, \lambda_{i}^{+} \geq 0(i=1 \cdots n)$ and the objective function

$$
D\left(p^{-}, p^{+}\right)+\sum_{i=1}^{n} \lambda_{i}^{-}\left(f\left(x_{i}, p^{-}\right)-y_{i}\right)+\sum_{i=1}^{n} \lambda_{i}^{+}\left(y_{i}-f\left(x_{i}, p^{+}\right)\right)
$$

(see for instance [51]). When $n$ is large, the determination of $2 n$ multipliers could be computationally heavy. This why we propose here another approach.

For each $p \in P$, we define the non-negative functions

$$
L(p)=\sum_{i=1}^{n} \min \left(y_{i}-f\left(x_{i}, p\right), 0\right)^{2}, \quad U(p)=\sum_{i=1}^{n} \max \left(y_{i}-f\left(x_{i}, p\right), 0\right)^{2} .
$$

Note that $L(p)=0$ amounts to say that the graph of the function $f(\cdot, p)$ is below $\mathcal{S}$. Similarly $U(p)=0$ amounts to say that the graph of the function $f(\cdot, p)$ is above $\mathcal{S}$. For a pair $\left(p^{-}, p^{+}\right)$, the sum $L\left(p^{-}\right)+U\left(p^{+}\right)$measures the quality of the framing of the set $\mathcal{S}$ by the graphs of the functions $f\left(\cdot, p^{-}\right)$and $f\left(\cdot, p^{+}\right)$, in the following sense. If a pair $\left(p^{-}, p^{+}\right)$satisfies

$$
L\left(p^{-}\right)+U\left(p^{+}\right) \leq \epsilon^{2}
$$


with $\epsilon \geq 0$, then one has

$$
f\left(x_{i}, p^{-}\right)-\epsilon \leq y_{i} \leq f\left(x_{i}, p^{+}\right)+\epsilon, \quad i=1 \cdots n
$$

For $\epsilon=0$, the constraint (10) is satisfied and we shall say that the frame is exact. For $\epsilon>0$, we shall say that the frame is $\epsilon$-approximate. In practice, one may be satisfied by an $\epsilon$-approximate frame, provided that $\epsilon$ is small enough. For a given $\epsilon>0$, one looks for the best $\epsilon$-approximate frames minimizing the function $D$, given by pairs $\left(p^{\star-}, p^{\star+}\right) \in P^{2}$ such that $D\left(p^{\star-}, p^{\star+}\right)=D_{\epsilon}^{\star}$ where

$$
D_{\epsilon}^{\star}:=\min \left\{D\left(p^{-}, p^{+}\right) ; L\left(p^{-}\right)+U\left(p^{+}\right) \leq \epsilon^{2}, ;\left(p^{-}, p^{+}\right) \in P^{2}\right\} .
$$

This is again a non-linear constrained optimization problem, that can be addressed with a penalty method, or with Karush-Kuhn-Tucker multipliers [51] (for this non-linear constraint, one may have also to deal with constraints qualification even when the set $P$ is described by a set of independent inequality constraints). Due to the particular structure of the problem, we propose a slightly different approach considering a multiplicative parameter of the cost and not of the constraint, as it is usually made. This brings some properties, as it will be shown in next Lemma 1 and Proposition 1, on which our method relies. Indeed, this approach brings also some flexibility in his application, that will be seen later in Section 3 and illustrated in Section 5. For a given positive number $\gamma$, we then consider the criterion

$$
J\left(p^{-}, p^{+}, \gamma\right):=L\left(p^{-}\right)+U\left(p^{+}\right)+\gamma D\left(p^{-}, p^{+}\right)
$$

and associate the optimization problem

$$
\left(\mathcal{P}_{\gamma}\right): \quad J^{\star}(\gamma):=\min \left\{J\left(p^{-}, p^{+}, \gamma\right) ;\left(p^{-}, p^{+}\right) \in P^{2}\right\} .
$$

Note that $\mathcal{P}_{\gamma}$ is an unconstrained problem, simpler to solve than problem (16), and we shall consider it for different values of $\gamma>0$. As the function $J$ is continuous w.r.t. $\left(p^{-}, p^{+}\right)$and $P$ is a compact set, the minimum in $J^{\star}(\gamma)$ exists for any $\gamma$.

We consider now assumptions guaranteeing the existence of non trivial frames of the data by functions in $\mathcal{C}$. First, we assume that the class $\mathcal{C}$ is rich enough to wrap all the data.

Assumption 2. There exists $\left(p^{-}, p^{+}\right) \in P^{2}$ such that (15) is fulfilled for $\epsilon=0$.

We shall consider cases for which all the data do not belong exactly to the graph of single function in $\mathcal{C}$ (otherwise the classical least-square method would do the job, providing best pairs with $p^{\star-}=p^{\star+}$ ).

Assumption 3. There does not exist $p \in P$ such that one has $y_{i}=f\left(x_{i}, p\right)$ for any $i \in\{1, \cdots, n\}$.

Let us first show that the optimization problem (18) generalizes the classical least-square method in the following way. Denote the classical least-square criterion by

$$
J_{L S}(p):=\sum_{i=1}^{n}\left(y_{i}-f\left(x_{i}, p\right)\right)^{2}
$$

and define the number

$$
J_{L S}^{\star}:=\min \left\{J_{L S}(p) ; p \in P\right\} .
$$


Lemma 1. Under Assumptions (1)-(2)-(3), one has

$$
\lim _{\gamma \rightarrow+\infty} J^{\star}(\gamma)=J_{L S}^{\star}
$$

Proof. Notice that $\gamma \mapsto J^{\star}(\gamma)$ is non decreasing, as the family of functions $(J(\cdot, \cdot, \gamma))$ is non-decreasing w.r.t. $\gamma$, and is bounded from above by $J_{L S}^{\star}$. Therefore the limit of $J^{\star}(\gamma)$ when $\gamma$ tends to $+\infty$ exists.

Consider a sequence of optimal pairs $\left(p_{n}^{\star-}, p_{n}^{\star+}\right)$ for $\gamma=n$. As $P$ is compact, the sequence converges when $n \rightarrow+\infty$, up to a sub-sequence, to a certain $\left(p_{\infty}^{\star-}, p_{\infty}^{\star+}\right) \in P^{2}$ such that $J\left(p_{\infty}^{\star-}, p_{\infty}^{\star+}, \infty\right)=\lim _{\gamma \rightarrow+\infty} J^{\star}(\gamma)$. This implies that one has necessarily $D\left(p_{\infty}^{\star-}, p_{\infty}^{\star+}\right)=0$, that is $f\left(x_{i}, p_{\infty}^{\star-}\right)=f\left(x_{i}, p_{\infty}^{\star+}\right)$ for any $i=1 \cdots n$. Consequently, one has $J\left(p_{\infty}^{\star-}, p_{\infty}^{\star+}, \infty\right) \geq J_{L S}^{\star}$ and we obtain that equality (19) is verified.

Remark 2. It may happen that the data set $\mathcal{S}$ is too poor or that the class $\mathcal{C}$ is over-parameterized. We shall say that $\mathcal{S}$ is rich enough for $\mathcal{C}$ when the property

$$
\left\{f\left(x_{i}, p_{a}\right)=f\left(x_{i}, p_{b}\right) ; i=1 \cdots n\right\} \Rightarrow p_{a}=p_{b}
$$

is fulfilled (which amounts to require the model $\{y=f(x, p) ; p \in P\}$ to be identifiable). Under this last condition, any converging sequence of optimal pairs $\left(p_{n}^{\star-}, p_{n}^{\star+}\right)$ for $\gamma_{n}$ satisfies $p_{\infty}^{\star-}=p_{\infty}^{\star+}$, i.e. the optimal frames converge when $\gamma \rightarrow+\infty$ to single curves, solutions of the least-square optimization.

We give now properties on which the method we propose to obtain functional framing is based.

Proposition 1. Under Assumptions 1-2-3, consider for any $\epsilon>0$ the set

$$
\Gamma_{\epsilon}:=\left\{\gamma \geq 0 ; Q_{\epsilon} \neq \emptyset\right\}
$$

where

$$
Q_{\epsilon}(\gamma):=\left\{\left(p^{-}, p^{+}\right) \in P^{2} ; J^{\star}(\gamma)=J\left(p^{-}, p^{+}, \gamma\right), L\left(p^{-}\right)+U\left(p^{+}\right) \leq \epsilon^{2}\right\}
$$

Then, the following properties are satisfied for any $\epsilon>0$.

1. $\Gamma_{\epsilon}$ is a closed interval $\left[0, \gamma_{\epsilon}^{\star}\right]$ with $0<\gamma_{\epsilon}^{\star}<+\infty$.

2. For any pair $\left(p^{\star-}, p^{\star+}\right)$ in $Q_{\epsilon}\left(\gamma_{\epsilon}^{\star}\right)$, one has

$$
D\left(p^{\star-}, p^{\star+}\right) \leq D\left(p^{-}, p^{+}\right), \forall\left(p^{-}, p^{+}\right) \in Q_{\epsilon}(\gamma), \forall \gamma<\gamma_{\epsilon}^{\star} .
$$

3. For any pair $\left(p^{\star-}, p^{\star+}\right)$ in $Q_{\epsilon}\left(\gamma_{\epsilon}^{\star}\right)$, one has the estimate

$$
D\left(p^{\star-}, p^{\star+}\right)-D_{\epsilon}^{\star} \leq \frac{\epsilon^{2}-L\left(p^{\star-}\right)-U\left(p^{\star+}\right)}{\gamma_{\epsilon}^{\star}} .
$$

Moreover, if there exists an unique pair $\left(p^{\star-}, p^{\star+}\right)$ realizing the minimum of $J\left(p^{-}, p^{+}, \gamma\right)$ among $\left(p^{-}, p^{+}\right)$in $P^{2}$, then one has necessarily $D\left(p^{\star-}, p^{\star+}\right)=D_{\epsilon}^{\star}$ with $L\left(p^{\star-}\right)+U\left(p^{\star+}\right)=\epsilon^{2}$. 
Proof. Let $\gamma_{1}, \gamma_{2}$ be two positive numbers with $\gamma_{1}<\gamma_{2}$. Consider pairs $\left(p_{1}^{-}, p_{1}^{+}\right)$, $\left(p_{2}^{-}, p_{2}^{+}\right)$such that $J^{\star}\left(\gamma_{1}\right)=J\left(p_{1}^{-}, p_{1}^{+}, \gamma_{1}\right)$ and $J^{\star}\left(\gamma_{2}\right)=J\left(p_{2}^{-}, p_{2}^{+}, \gamma_{2}\right)$. One can write

$$
\begin{aligned}
J^{\star}\left(\gamma_{2}\right)=J\left(p_{2}^{-}, p_{2}^{+}, \gamma_{2}\right) & \leq J\left(p_{1}^{-}, p_{1}^{+}, \gamma_{2}\right) \\
& =J^{\star}\left(\gamma_{1}\right)+\left(\gamma_{2}-\gamma_{1}\right) D\left(p_{1}^{-}, p_{1}^{+}\right) \\
& \leq J\left(p_{2}^{-}, p_{2}^{+}, \gamma_{1}\right)+\left(\gamma_{2}-\gamma_{1}\right) D\left(p_{1}^{-}, p_{1}^{+}\right)
\end{aligned}
$$

from which one obtains

$$
J\left(p_{2}^{-}, p_{2}^{+}, \gamma_{2}\right)-J\left(p_{2}^{-}, p_{2}^{+}, \gamma_{1}\right) \leq\left(\gamma_{2}-\gamma_{1}\right) D\left(p_{1}^{-}, p_{1}^{+}\right)
$$

or equivalently

$$
\left(\gamma_{2}-\gamma_{1}\right) D\left(p_{2}^{-}, p_{2}^{+}\right) \leq\left(\gamma_{2}-\gamma_{1}\right) D\left(p_{1}^{-}, p_{1}^{+}\right) .
$$

This shows the following property

$$
\left.\begin{array}{c}
\gamma_{1}<\gamma_{2} \\
J^{\star}\left(\gamma_{1}\right)=J\left(p_{1}^{-}, p_{1}^{+}, \gamma_{1}\right) \\
J^{\star}\left(\gamma_{2}\right)=J\left(p_{2}^{-}, p_{2}^{+}, \gamma_{2}\right)
\end{array}\right\} \Rightarrow D\left(p_{1}^{-}, p_{1}^{+}\right) \geq D\left(p_{2}^{-}, p_{2}^{+}\right)
$$

that shall be useful in the following.

For convenience, let us define the sub-sets

$$
Q_{\epsilon}:=\left\{\left(p^{-}, p^{+}\right) \in P^{2} ; L\left(p^{-}\right)+U\left(p^{+}\right) \leq \epsilon^{2}\right\} .
$$

Thanks to Assumptions 1 and 2, $Q_{\epsilon}$ is a non-empty compact set for any nonnegative $\epsilon$ (including 0 ). Then the numbers

$$
D_{\epsilon}^{\star}:=\min \left\{D\left(p^{-}, p^{+}\right) ;\left(p^{-}, p^{+}\right) \in Q_{\epsilon}\right\}
$$

are well defined. Moreover, by Assumption $3, D_{\epsilon}^{\star}$ is positive for any $\epsilon \geq 0$.

1. We begin by showing that for any $\epsilon>0$, the set $\Gamma_{\epsilon}$ contains at least one positive element. For any $\epsilon>0$, consider $\gamma=\epsilon^{2} / D_{0}^{\star}$ and $\left(p_{0}^{-}, p_{0}^{+}\right) \in Q_{0}$ (i.e. such that $\left.D\left(p_{0}^{-}, p_{0}^{+}\right)=D_{0}^{\star}\right)$. One has

$$
J^{\star}(\gamma) \leq J\left(p_{0}^{-}, p_{0}^{+}, \gamma\right)=\gamma D_{0}^{\star}=\epsilon^{2}
$$

and any pair $\left(p^{-}, p^{+}\right)$realizing $J^{\star}(\gamma)$ verifies then

$$
L\left(p^{-}\right)+U\left(p^{+}\right)+\gamma D\left(p^{-}, p^{+}\right) \leq \epsilon \quad \Rightarrow \quad L\left(p^{-}\right)+U\left(p^{+}\right) \leq \epsilon^{2}
$$

that is $\left(p^{-}, p^{+}\right) \in Q_{\epsilon}(\gamma)$, from which we deduce that $\gamma$ belongs to $\Gamma_{\epsilon}$.

We show now that $\Gamma_{\epsilon}$ is an interval containing 0 . Let $\gamma>0$ belonging to $\Gamma_{\epsilon}$ and $\left(p^{-}, p^{+}\right)$a pair realizing $J^{\star}(\gamma)$ with $L\left(p^{-}\right)+U\left(p^{+}\right) \leq \epsilon^{2}$. Take $\gamma^{\prime} \in[0, \gamma)$ and let $\left(p^{\prime-}, p^{\prime+}\right)$ be a pair realizing $J^{\star}\left(\gamma^{\prime}\right)$. One can write

$$
\begin{aligned}
J^{\star}\left(\gamma^{\prime}\right)=L\left(p^{-}\right)+U\left(p^{+}\right)+\gamma^{\prime} D\left(p^{-}, p^{\prime+}\right) & \leq L\left(p^{-}\right)+U\left(p^{+}\right)+\gamma^{\prime} D\left(p^{-}, p^{+}\right) \\
& \leq \epsilon^{2}+\gamma^{\prime} D\left(p^{-}, p^{+}\right)
\end{aligned}
$$


or equivalently

$$
L\left(p^{\prime-}\right)+U\left(p^{\prime+}\right) \leq \epsilon^{2}+\gamma^{\prime}\left(D\left(p^{-}, p^{+}\right)-D\left(p^{\prime-}, p^{++}\right)\right) .
$$

From property (24), we have $D\left(p^{-}, p^{+}\right) \leq D\left(p^{-\prime}, p^{+\prime}\right)$, which demonstrates that the pair $\left(p^{-\prime}, p^{+\prime}\right)$ belongs to $Q_{\epsilon}\left(\gamma^{\prime}\right)$, that is $\gamma^{\prime}$ belongs to $\Gamma_{\epsilon}$.

Now we show that the upper bound $\gamma_{\epsilon}^{\star}=\sup \Gamma_{\epsilon}$ belongs to $\Gamma_{\epsilon}$. Let $\gamma_{n}$ be an increasing sequence converging to $\gamma_{\epsilon}^{\star}$ and $\left(p_{n}^{-}, p_{n}^{+}\right)$a sequence of pairs in $Q_{\epsilon}$ realizing $J^{*}\left(\gamma_{n}\right)$. For any $\left(p^{-}, p^{+}\right) \in P^{2}$, one has

$$
J\left(p_{n}^{-}, p_{n}^{+}, \gamma_{n}\right) \leq J\left(p^{-}, p^{+}, \gamma_{n}\right), \quad \forall n .
$$

As $Q_{\epsilon}$ is a compact set, there exists a sub-sequence, also noted $\left(p_{n}^{-}, p_{n}^{+}\right)$, which converges to a certain $\left(p^{\star-}, p^{\star+}\right)$ in $Q_{\epsilon}$. By continuity of the function $J$, one obtains that the inequality

$$
J\left(p^{\star-}, p^{\star+}, \gamma_{\epsilon}^{\star}\right) \leq J\left(p^{-}, p^{+}, \gamma_{\epsilon}^{\star}\right)
$$

is fulfilled for any $\left(p^{-}, p^{+}\right) \in P^{2}$, which shows that the pair $\left(p^{\star-}, p^{\star+}\right)$ realizes $J\left(\gamma_{\epsilon}^{\star}\right)$, and thus $\gamma_{\epsilon}^{\star}$ belongs to $\Gamma_{\epsilon}$. This proves the point 1 .

2. The point 2 . is a direct consequence of the property (24).

3. Consider a pair $\left(p_{\epsilon}^{-}, p_{\epsilon}^{+}\right)$in $P^{2}$ such that $D\left(p_{\epsilon}^{-}, p_{\epsilon}^{+}\right)=D_{\epsilon}^{\star}$. One has then

$$
J^{\star}\left(\gamma_{\epsilon}^{\star}\right) \leq J\left(p_{\epsilon}^{-}, p_{\epsilon}^{+}, \gamma_{\epsilon}^{\star}\right)=\epsilon^{2}+\gamma_{\epsilon}^{\star} D_{\epsilon}^{\star}
$$

Therefore, any pair $\left(p^{\star-}, p^{\star+}\right)$ in $Q_{\epsilon}\left(\gamma_{\epsilon}^{\star}\right)$ verifies

$$
L\left(p^{\star-}\right)+U\left(p^{\star+}\right)+\gamma_{\epsilon}^{\star} D\left(p^{\star-}, p^{\star+}\right) \leq \epsilon^{2}+\gamma_{\epsilon}^{\star} D_{\epsilon}^{\star}
$$

or equivalently the inequality (23).

Take a decreasing sequence $\gamma_{n}$ converging to $\gamma_{\epsilon}^{\star}$, and pairs $\left(p_{n}^{-}, p_{n}^{+}\right)$in $P^{2}$ such that $J^{\star}\left(\gamma_{n}\right)=J\left(p_{n}^{-}, p_{n}^{+}, \gamma_{n}\right)$ for any $n$. One has necessarily $L\left(p_{n}^{-}\right)+$ $U\left(p_{n}^{+}\right)>\epsilon$ and can write

$$
J^{\star}\left(\gamma_{n}\right)=L\left(p_{n}^{-}\right)+U\left(p_{n}^{+}\right)+\gamma_{n} D\left(p_{n}^{-}, p_{n}^{+}\right) \leq J\left(p_{\epsilon}^{-}, p_{\epsilon}^{+}, \gamma_{n}\right) \leq \epsilon^{2}+\gamma_{n} D_{\epsilon}^{\star}
$$

or equivalently

$$
\gamma_{n}\left(D\left(p_{n}^{-}, p_{n}^{+}\right)-D_{\epsilon}^{\star}\right) \leq \epsilon^{2}-\left(L\left(p_{n}^{-}\right)+U\left(p_{n}^{+}\right)\right)<0 .
$$

Therefore, one has $D\left(p_{n}^{-}, p_{n}^{+}\right)<D_{\epsilon}^{\star}$ for any $n$. Consider a sub-sequence of $\left(p_{n}^{-}, p_{n}^{+}\right)$converging to a certain $\left(p^{\star-}, p^{\star+}\right)$. It necessarily satisfies $L\left(p^{\star-}\right)+$ $U\left(p^{\star-}\right) \geq \epsilon^{2}$ and $D\left(p^{\star-}, p^{\star+}\right) \leq D_{\epsilon}^{\star}$ by continuity of the functions $L, U$ and $D$. As previously, one can show that $\left(p^{\star-}, p^{\star+}\right)$ is a maximizer of $J^{\star}\left(\gamma_{\epsilon}^{\star}\right)$. As we know that there exists a maximizer of $J^{\star}\left(\gamma_{\epsilon}^{\star}\right)$ in $Q_{\epsilon}$, we deduce that when the maximizer of $J^{\star}\left(\gamma_{\epsilon}^{\star}\right)$ is unique, it has to verify $L\left(p^{\star-}\right)+U\left(p^{\star-}\right)=\epsilon^{2}$ and by property $(23)$, its has also to realize $D\left(p^{\star-}, p^{\star+}\right)=D_{\epsilon}^{\star}$.

The properties of the set $\Gamma_{\epsilon}$ given in Proposition (1) leads to the following method for the search of tight $\epsilon$-approximate frames. 
Proposition 2. Consider that Assumptions 1-2-3 are fulfilled. Take $\epsilon>0$ such that $\left.\epsilon<\frac{J_{L S}^{\star}}{\sqrt{n} M}\right)$, where $M:=\max _{i=1 \cdots n} \max _{p \in P}\left|y_{i}-f\left(p, x_{i}\right)\right|$, and define the number

$$
\bar{\gamma}_{\epsilon}=\frac{1}{2} \frac{J_{L S}^{\star}}{J_{L S}^{\star}-\sqrt{n} M \epsilon}
$$

Then the procedure of a dichotomy search

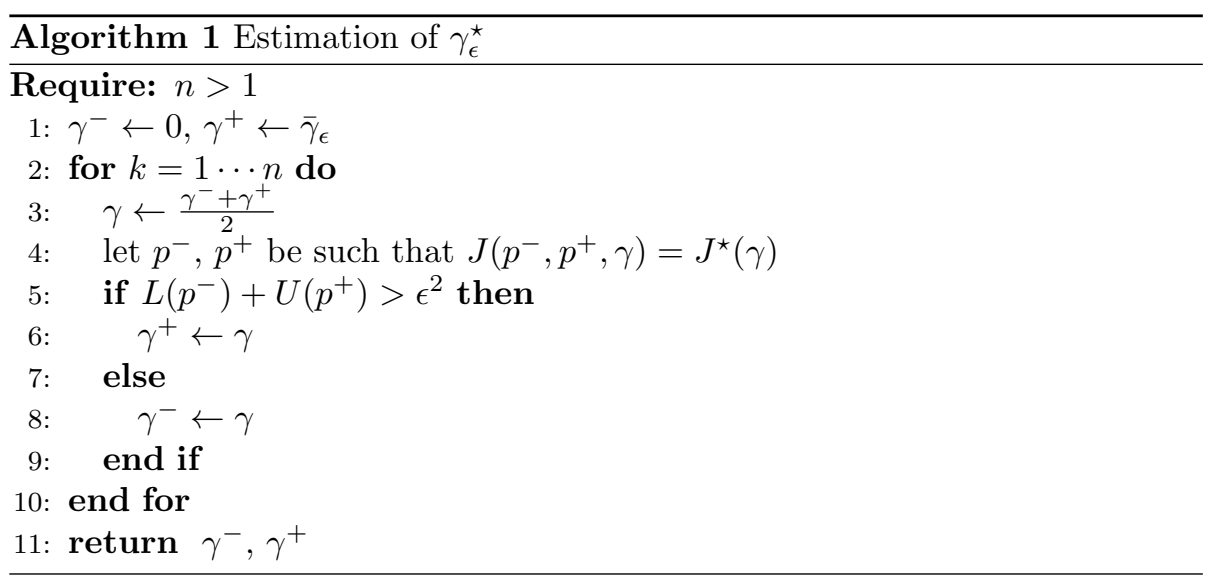

provides an estimation of $\gamma_{\epsilon}^{\star} \in\left[\gamma^{-}, \gamma^{+}\right]$with $\gamma^{+}-\gamma^{-}<2^{-n} \bar{\gamma}_{\epsilon}$.

Proof. Let us show that $\bar{\gamma}_{\epsilon}$ is an upper bound of the number $\gamma_{\epsilon}^{\star}$ given by Proposition (1). Remark first that having $p^{-}=p^{+}=p$ gives $J(p, p, \gamma)=J_{L S}(p)$ whatever is $\gamma$. Let $\tilde{p}$ be a solution of the least-square fitting, that is $\tilde{p}$ satisfying $J_{L S}(\tilde{p})=J_{L S}^{\star}$. As $\mathcal{S}$ does not belong to the graph of a single function in $\mathcal{C}$ (Assumption 3), one has $J_{L S}^{\star}>0$. Let $\gamma \in \Gamma_{\epsilon}$ and $\left(p^{-}, p^{+}\right) \in Q_{\epsilon}(\gamma)$. One can write

$$
\begin{aligned}
J_{L S}^{\star}=J(\tilde{p}, \tilde{p}, \gamma) & \geq J^{\star}(\gamma)=J\left(p^{-}, p^{+}, \gamma\right) \\
& \geq \gamma D\left(p^{-}, p^{+}\right) \\
& =\gamma \sum_{i=1}^{n}\left(\left(f\left(x_{i}, p^{+}\right)-y_{i}\right)+\left(y_{i}-f\left(x_{i}, p^{-}\right)\right)\right)^{2} \\
& =\gamma \sum_{i=1}^{n}\left(y_{i}-f\left(x_{i}, p^{+}\right)\right)^{2}+\left(y_{i}-f\left(x_{i}, p^{-}\right)\right)^{2} \\
& +2\left(f\left(x_{i}, p^{+}\right)-y_{i}\right)\left(y_{i}-f\left(x_{i}, p^{-}\right)\right)
\end{aligned}
$$

and thus

$$
\begin{aligned}
& J_{L S}^{\star} \geq \gamma\left(J_{L S}\left(p^{-}\right)+\right.\left.J_{L S}\left(p_{n}^{+}\right)\right) \\
& \quad+2 \gamma \sum_{i=1}^{n}\left(f\left(x_{i}, p^{+}\right)-y_{i}\right)\left(y_{i}-f\left(x_{i}, p^{-}\right)\right) \\
& \geq 2 \gamma\left(J_{L S}^{\star}+\sum_{i=1}^{n}\left(f\left(x_{i}, p^{+}\right)-y_{i}\right)\left(y_{i}-f\left(x_{i}, p^{-}\right)\right)\right) .
\end{aligned}
$$

In this last summation, consider the subset $I$ of indices $i$ for which

$$
\left(f\left(x_{i}, p^{+}\right)-y_{i}\right)\left(y_{i}-f\left(x_{i}, p^{-}\right)\right)<0 .
$$


For $i \in I$, let $\eta_{i}=\max \left(y_{i}-f\left(x_{i}, p^{+}\right), 0\right)+\max \left(f\left(x_{i}, p^{-}\right)-y_{i}, 0\right)$ and one has then

$$
\left(f\left(x_{i}, p^{+}\right)-y_{i}\right)\left(y_{i}-f\left(x_{i}, p^{-}\right)\right)>-M \eta_{i}
$$

where the numbers $\eta_{i}$ satisfy $\sum_{i \in I} \eta_{i}^{2} \leq \epsilon^{2}$. One obtains then the lower bound

$$
\sum_{i=1}^{n}\left(f\left(x_{i}, p^{+}\right)-y_{i}\right)\left(y_{i}-f\left(x_{i}, p^{-}\right)\right) \geq-M \sum_{i \in I} \eta_{i} \geq-M \sqrt{n} \epsilon
$$

from which one deduces that the number $\gamma$ has to satisfy the inequality

$$
J_{L S}^{\star} \geq 2 \gamma\left(J_{L S}^{\star}-\sqrt{n} M \epsilon\right)
$$

or equivalently $\gamma \leq \bar{\gamma}_{\epsilon}$.

Finally, due to the property of $\Gamma_{\epsilon}$ being an interval $\left[0, \gamma_{\epsilon}^{\star}\right]$ (cf Proposition 1), the dichotomous algorithm 1 converges to $\gamma_{\epsilon}^{\star}$ with a guaranteed lower bound at any iteration step.

Our method consists then in an iteration of the unconstrained problem $\mathcal{P}_{\gamma}$ for different positive values of $\gamma$ to obtain an approximation of $\gamma_{\epsilon}^{\star}$, accordingly to Proposition 2. Note that Proposition 1 guarantees the positivity of $\gamma_{\epsilon}^{\star}$ only when $\epsilon$ is positive. Our method is thus fundamentally based on $\epsilon$-approximate frames (for $\epsilon=0$, one may have $\gamma_{0}^{\star}=0$ and all frames realize $J^{\star}(0)=0$ ). In practice, for a small positive value of $\epsilon$, the algorithm starts with an upper bound $\bar{\gamma}_{\epsilon}$ close to $1 / 2$ and a few iterations are enough to obtain an accurate approximation of the value of $\gamma_{\epsilon}^{\star}$ for a reasonably small value of $\epsilon$. For this approximate value of $\gamma_{\epsilon}^{\star}$, one then gets from the optimization problem $\mathcal{P}_{\gamma_{\epsilon}^{\star}}$ a maximizer $\left(p^{\star-}, p^{\star+}\right)$. If $L\left(p^{\star-}\right)+U\left(p^{\star+}\right)=\epsilon^{2}$, then the frame is optimal (i.e. $\left.D\left(p^{\star-}, p^{\star+}\right)=D_{\epsilon}^{\star}\right)$, accordingly to Proposition 1 . If the optimal solution of $\mathcal{P}_{\gamma_{\epsilon}^{\star}}$ is not unique, one may have $L\left(p^{\star-}\right)+U\left(p^{\star+}\right)<\epsilon^{2}$ but expression (23) of Proposition 1 gives then an estimation of its sub-optimality. Note that the method is robust with respect to the performances of the optimization technique used to solve the problem $\mathcal{P}_{\gamma}$ or the choice of the number of iterations in the algorithm. If it provides a suboptimal solution (for the value of $D(\cdot)$ ), it always generate an $\epsilon$-approximate frame. We shall see on concrete cases in Section 5 that this method turns out to be fast and efficient, with a simple implementation.

Remark 3. For any pair $\left(p^{-}, p^{+}\right)$in $Q_{\epsilon}\left(\gamma_{\epsilon}^{\star}\right)$, we can also measure the width of the frame by the area delimited by the graphs of the functions $f\left(\cdot, p^{+}\right)$and $f\left(\cdot, p^{-}\right)$:

$$
A\left(p^{-}, p^{+}\right):=\int_{\min _{i} x_{i}}^{\max _{i} x_{i}}\left(f\left(x, p^{+}\right)-f\left(x, p^{-}\right)\right) d x .
$$

This allows one to associate to the class of functions $\mathcal{C}$ an "adequacy" value

$$
V_{\epsilon}(\mathcal{C}):=\min \left\{A\left(p^{-}, p^{+}\right) ;\left(p^{-}, p^{+}\right) \in Q_{\epsilon}\left(\gamma_{\epsilon}^{\star}\right)\right\}
$$

and to compare the adequacy of each class. For two classes $\mathcal{C}_{1}, \mathcal{C}_{2}$ of functions, we shall say that $\mathcal{C}_{1}$ gives a better $\epsilon$-approximate framing of the set $\mathcal{S}$ than the class $\mathcal{C}_{2}$ when one has $V_{\epsilon}\left(\mathcal{C}_{1}\right)<V_{\epsilon}\left(\mathcal{C}_{2}\right)$. This will be illustrated in Section 5. 


\section{Extensions of the method}

The extensions we present here bring some additional flexibility to the method, in terms of choice of the class of functions and width of frames. For simplicity of the presentation, we have not incorporated them in the presentation of the overall method. Both extensions are illustrated in Section 5.

\subsection{Framing with different upper and lower classes}

There is no a priori reason to impose that upper and lower functions in the frame (10) are sought among the same class of functions. Typically, one may face growth inhibition or not, depending on the experiments. One can then choose a family of uninhibited growth models for the upper class, and inhibited ones for the lower class (this is illustrated in Section 5 with Monod and Haldane functions). Therefore, we can consider without loss of generality two classes

$$
\mathcal{C}^{-}:=\bigcup_{p \in P^{-}}\left\{f^{-}(\cdot, p): \mathbb{R} \mapsto \mathbb{R}\right\}, \quad \mathcal{C}^{+}:=\bigcup_{p \in P^{+}}\left\{f^{+}(\cdot, p): \mathbb{R} \mapsto \mathbb{R}\right\}
$$

instead of the single class $\mathcal{C}$ considered in (9). Here, $P^{-}$and $P^{+}$are two subsets of $\mathbb{R}^{m^{-}}$and $\mathbb{R}^{m^{+}}$, such that Assumption 1 is fulfilled for $P^{-}, f^{-}$, and $P^{+}, f^{+}$. The dimension $\mathrm{m}^{-}$is not necessarily equal to $\mathrm{m}^{+}$. In Section 5 , we shall see that this allows one to consider less parameters altogether.

In short, we do not give here the corresponding assumptions and statement, as it simply consists in replacing $P \times P$ by $P^{-} \times P^{+}$in Assumption 2 and Propositions 1, 2 of Section 2. We shall also consider that Assumption 3 is verified for both $P^{-}$and $P^{+}$.

\subsection{Relaxing with one or more points out of the frame}

In practice, the frames provided by Proposition 1 might be too conservative as it considers all the experimental data. Practitioners might want to have tightened bounds, allowing to have one or several points unwrapped. They could of course choose themselves which point(s) to be withdrawn and relaunch the method on a smaller data set. We show here how to extend in a simple way the method to have this done in a systematic way, i.e. as an automatic method choosing which data point(s) can be forgotten to obtain tightened bounds, depending on the maximal number of points that one accepts to be unwrapped. The idea of relaxing with one or more points out of the frame has been also considered in algorithms such as GOMNE [20] (see also Section 6.3.3 in [18]).

Let $j \in\{1, \cdots, n-1\}$ and denote by $\mathcal{I}_{n}^{j}$ the set of subsets $I \subset\{1, \cdots, n\}$ with card $I \leq j$. As an extension of the optimization problem (16) considered in Section 2, we consider the family of problems for $j=1 \cdots n-1$

$$
D_{\epsilon}^{\star j}:=\min \left\{D^{I}\left(p^{-}, p^{+}\right) ; L^{I}\left(p^{-}\right)+U^{I}\left(p^{+}\right) \leq \epsilon^{2},\left(p^{-}, p^{+}\right) \in P^{2}, I \in \mathcal{I}_{n}^{j}\right\}
$$

where

$$
D^{I}\left(p^{-}, p^{+}\right):=\sum_{i \notin I}\left(f\left(x_{i}, p^{+}\right)-f\left(x_{i}, p^{-}\right)\right)^{2}
$$


and

$$
L^{I}(p):=\sum_{i \notin I} \min \left(y_{i}-f\left(x_{i}, p\right), 0\right)^{2}, U^{I}(p):=\sum_{i \notin I} \max \left(y_{i}-f\left(x_{i}, p\right), 0\right)^{2} .
$$

When $n$ is large, these are computationally heavy problems, due to the combinatorics of $\mathcal{I}_{n}^{j}$. Instead, we propose to simply relax the constraint (14) by

$$
\exists I \in \mathcal{I}_{n}^{j}, \quad L^{I}\left(p^{-}\right)+U^{I}\left(p^{+}\right) \leq \epsilon^{2}
$$

while keeping the same criterion (18) defined with all $i \in\{1 \cdots n\}$. We then look for the largest $\gamma$ such that a solution $\left(p_{j}^{-}, p_{j}^{+}\right)$of problem $\mathcal{P}_{\gamma}$ satisfies the constraint (27) for a given $j>0$. Of course, this optimization problem does not guarantee that the pair $\left(p_{j}^{-}, p_{j}^{+}\right)$provides the minimal value of $D_{\epsilon}^{\star j}$, differently to what is proved in Proposition 1 for the set $Q_{\epsilon}\left(\gamma_{\epsilon}^{\star}\right)$. However, we shall see that this is a simple and effective method to obtain tight frames excluding $j$ data points (although non-optimal). This leads to the Algorithm 2 below (simply adding the step 5 below).

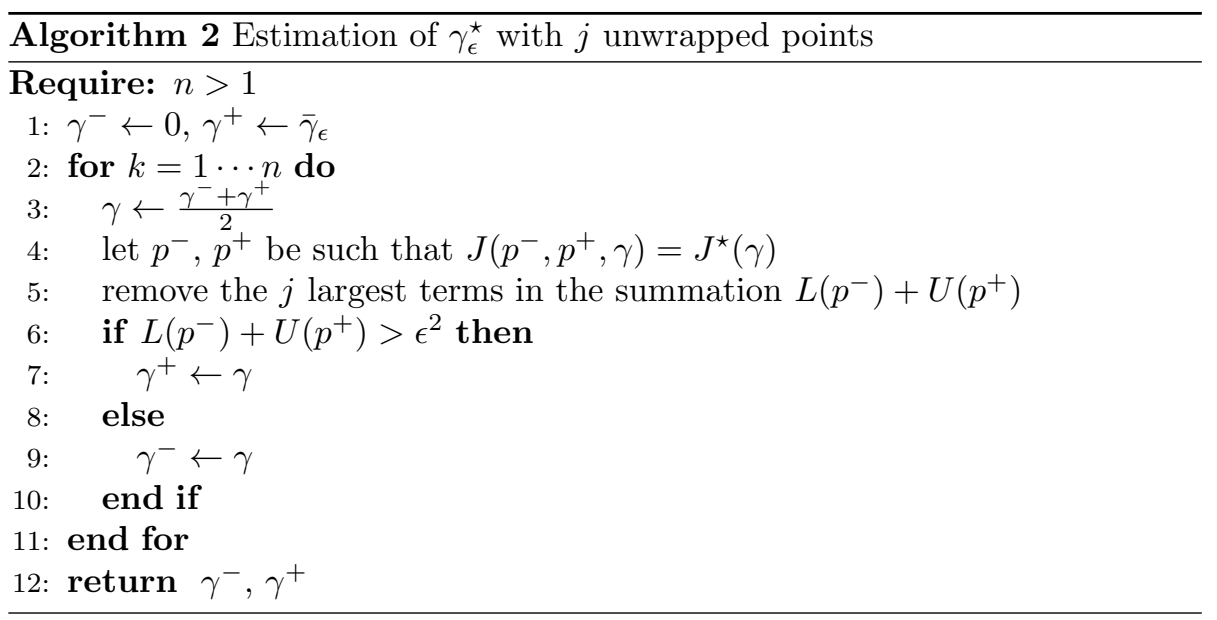

Then pairs $\left(p_{j}^{-}, p_{j}^{+}\right)$obtained with $j$ unwrapped points provide then a sensitivity of the framing with respect to the number of unwrapped points. Typically, one may choose the number $j$ of unwrapped points depending on the gain on the value $D\left(p_{j}^{-}, p_{j}^{+}\right)$which measures the frame width. This will be illustrated in Section 5.

\section{Application to the framing of growth curves}

The knowledge of the growth functions is very useful in microbiology to predict the evolution of micro-organisms and the performances of the bioprocesses, with models such as (6):

$$
\left\{\begin{aligned}
\dot{x} & =\mu(s) x-D x \\
\dot{s} & =-\frac{1}{r} \mu(s) x+D\left(s_{i n}-s\right)
\end{aligned}\right.
$$

where $x$ and $s$ stand here for the biomass and substrate concentrations, respectively. Typically, experiments to determine the unknown growth function $\mu(\cdot)$ are drawn as follows. 
- either in batch culture i.e. with $D=0$. Experiments consist in choosing different values $s_{i}$ of initial substrate concentration $s(0)=s_{i}$ and computing the value $\mu_{i}=\left.\frac{d}{d t} \ln (x(t))\right|_{t=0}$, measuring the time variation of $x(\cdot)$. Data points $\left(s_{i}, \mu_{i}\right)$ are then expected to belong to the graph of the unknown function $\mu(\cdot)$.

- either in continuous culture i.e. with $D>0$ (assumed to be constant or slowly varying). Experiments consist in choosing different values $D_{i}$ of $D$ and wait the system to be at a quasi-steady state $\left(x^{\star}, s^{\star}\right)$. Then, the data point $\left(s_{i}, \mu_{i}\right)=\left(s^{\star}, D_{i}\right)$ is expected to belong to the graph of the unknown function $\mu(\cdot)$.

While the chemostat experiments are quite time-consuming (but various experiments conducted in the past under different conditions can be collected together, which is in the spirit of the present approach), batch cultures can be easily performed in microwell plate with automatic measurements, so that a reasonably rich data set can be acquired. With the set of data points $\left(s_{i}, \mu_{i}\right)$, one usually conducts a non-linear regression to adjust the parameters of a growth model with least-square criterion [50], such as for instance the Monod growth function (8), or the Haldane one

$$
\mu(s)=\frac{\hat{\mu} s}{K+s+s^{2} / K_{i}} .
$$

describing the effect of growth inhibition for large concentrations of substrate. However, it has been underlined that the usual R-squared determination coefficient is not appropriate to measure the quality of the adjustment in this non-linearity context [48]. More importantly, reproducibility and environmental fluctuations (such as $\mathrm{pH}$, temperature, light...) from one experiment to another one are common issues in microbiology.

Here, we shall consider that the experiments have been sufficiently numerous to have faced the various possible kinds of fluctuations, so that future utilization of the micro-organisms might be subject to similar fluctuations or combinations of them occurring at various unknown times (this hypothesis is important to justify the framing approach, that we illustrate on synthetic data in the next section).

Let us now show how one can use the lower and upper functions $\mu^{-}(\cdot), \mu^{+}(\cdot)$ to obtain guaranteed predictions of $x(\cdot)$ and $s(\cdot)$. Assume that yield coefficient $r$, dilution rate $D$ (possibly time-varying) and initial condition $\left(x_{0}, s_{0}\right)$ of model (28) are known. Then, one can easily show that the variable $z(t)=x(t)+r s(t)$ is solution of $\dot{z}=D\left(r s_{i n}-z\right)$. Therefore, one has

$$
z(t)=r s_{i n}+\left(x_{0}+r s_{0}-r s_{i n}\right) e^{-D t}, \quad t \geq 0 .
$$

We distinguish now the predictions of $x(t)$ and $s(t)$.

1. Prediction of $x(t)$. From $s(t)=\frac{1}{r}(z(t)-x(t))$, one obtains that $x(\cdot)$ is solution of the (non-autonomous) scalar differential equation:

$$
\dot{x}=\mu\left(\frac{1}{r}(z(t)-x)\right) x-D x
$$


Then, following [56, Th. VIII, p. 95], a frame $x^{-}(t) \leq x(t) \leq x^{+}(t)$ is obtained for any $t \geq 0$ when $x^{-}(\cdot), x^{+}(\cdot)$ are solutions of

$$
\left\{\begin{array}{l}
\dot{x}^{-}=\mu^{-}\left(\frac{1}{r}\left(z(t)-x^{-}\right)\right) x^{-}-D x^{-}, x^{-}(0)=x_{0} \\
\dot{x}^{+}=\mu^{+}\left(\max \left(\frac{1}{r}\left(z(t)-x^{+}\right), 0\right)\right) x^{+}-D x^{+}, x^{+}(0)=x_{0}
\end{array}\right.
$$

2. Prediction of $s(t)$. Similarly, one has $x(t)=z(t)-r s(t)$ and $s(\cdot)$ solution of the scalar dynamics

$$
\dot{s}=-\frac{1}{r} \mu(s)(z(t)-r s(t))+D\left(s_{i n}-s\right)
$$

which gives a frame $s^{-}(t) \leq s(t) \leq s^{+}(t)$ where $s^{-}(\cdot), s^{+}(\cdot)$ are solutions of

$$
\left\{\begin{array}{l}
\dot{s}^{-}=-\frac{1}{r} \mu^{+}\left(s^{-}\right)\left(z(t)-r s^{-}\right)+D\left(s_{i n}-s^{-}\right), s^{-}(0)=s_{0} \\
\dot{s}^{+}=-\frac{1}{r} \mu^{-}\left(s^{+}\right) \max \left(z(t)-r s^{+}, 0\right)+D\left(s_{i n}-s^{+}\right), s^{-}(0)=s_{0}
\end{array}\right.
$$

Remark that uncertainties on the initial condition $\left(x_{0}, s_{0}\right) \in\left[x_{0}^{-}, x_{0}^{+}\right] \times$ $\left[s_{0}^{-}, s_{0}^{+}\right]$can be also incorporated in the framing, considering

$z^{-}(t)=r s_{i n}+\left(x_{0}^{-}+r s_{0}^{-}-r s_{i n}\right) e^{-D t}, z^{+}(t)=r s_{i n}+\left(x_{0}^{+}+r s_{0}^{+}-r s_{i n}\right) e^{-D t}$

instead of $z(t)$ in systems (31)-(32) and initialization $x^{-}(0)=x_{0}^{-}, x^{+}(0)=x_{0}^{+}$, $s^{-}(0)=s_{0}^{-}, s^{+}(0)=s_{0}^{+}$.

\section{Numerical illustrations}

In this section, we test the framing method on numerical data and illustrate on synthetic data (for which the fluctuating trajectories are known) how the method provides state estimation with guaranteed intervals. We proceed as follows.

1. We first generate a set of growth curves $\mu$, by random sampling in the class of Monod (8) or Haldane (29) functions, accordingly to uniform distributions of their parameters on given intervals. This provides a random set $\mathcal{R}$ of parameters. Each curve represents the growth in a plausible environment.

2. For each growth curve $\mu$, we generate a sequence of points $\left(s_{i}, \mu_{i}\right)_{i}$ where $\left(s_{i}\right)_{i}$ is a random increasing sequence (with given bounds between two consecutive elements), and $\mu_{i}$ is the value $\mu\left(s_{i}\right)$ corrupted by a bounded uniform noise. The sequence $\left(s_{i}, \mu_{i}\right)_{i}$ represents a plausible set of measurements of an experiment in a fixed environment. Besides, we have added a small number of outliers that could represent measurement errors as this can happens punctually in biology. Outliers have been generated by uniform drawing outside the amplitude of considered noises. Gathering the sequences of points for all the generated curves provides a set $\mathcal{S}$ of "synthetic" data. 
3. We apply our framing method to the data set $\mathcal{S}$ for determining the best lower and upper functions $\mu^{-}(\cdot), \mu^{+}(\cdot)$ for a given $\epsilon$ accuracy (cf Proposition 1), with $\epsilon$ small.

4. We use the functions $\mu^{-}(\cdot), \mu^{+}(\cdot)$ to compute time varying intervals $\left[x^{-}(\cdot), x^{+}(\cdot)\right],\left[s^{-}(\cdot), s^{+}(\cdot)\right]$ for the concentrations $x(\cdot), s(\cdot)$, as solutions of equations (30)-(31)-(32).

5 . We simulate the dynamical model (28) where the function $\mu(\cdot)$ changes at random times following an exponential distribution. At each of these times, the function $\mu$ is drawn uniformly among functions (8) or (29) with parameters within $\mathcal{R}$. This represents plausible changes in environments with time. We then compare (in the transients) the solution $x(\cdot), s(\cdot)$ with the lower and upper estimates provided in the previous step.

We have also computed the average growth function (provided by the classical least squares method) and its corresponding trajectory to appreciate its position within the intervals. The optimization problem $\mathcal{P}_{\gamma}$ has been solved numerically with the fmincon function of Matlab software (without constraint). The bounds for the search of pairs $\left(p^{-}, p^{+}\right)$have been chosen

- for the Monod expression: with $\mu_{\max } \in[0.2,2], K_{s} \in[0,0.2]$, while the generated curves have been drawn keeping $\mu_{\max } \in[0.1,1.8]$ and $K_{s} \in$ $[0.1,0.2]$,

- for the Haldane expression: with $\bar{\mu} \in[0.2,2], K \in[0,0.2], K_{i} \in[0,6]$, while the generated curves have been drawn keeping $\bar{\mu} \in[0.1,1.8], K_{s} \in[0.1,0.2]$ and $K_{i} \in[0,10]$.

The maximal amplitude of noise has been chosen to be equal to $10 \%$ of the measurement value, and outliers have been generated within an interval three times larger. Outliers represent $10 \%$ of the data. For the simulations of random times of changes of the growth function, the rate parameter of the exponential distribution has been chosen to be 10 times the time horizon. The algorithm of Proposition 2 has been performed for $\epsilon=10^{-3}$, starting with an upper bound $\gamma^{+}=1 / 2$. Twenty iterations were more than enough to obtain a tight framing $\left(\epsilon=10^{-6}\right.$ with 40 iterations have tested and have given very similar results).

\subsection{Example of a batch process}

We have generated randomly ten growth curves (see Fig. 1, left) and one hundred data corrupted with noise (see Fig. 1-right).

We have wrapped all the data points among the class of Monod functions, using the method exposed in Section 2, which gives the functions $\mu^{-}, \mu^{+}$whose graphs are depicted on Fig. 2-left. In addition, the graph of the growth curve $\bar{\mu}$ determined by the usual least-square method is plotted in dashed line. To show the convergence of the method, Fig. 3 depicts the frames obtained for $\gamma^{+}$ at each iteration of Algorithm 1 of Proposition 2 (remind that $\gamma^{+}$represents an "inner" estimation of the frame). One can see that the frame obtained at the first iteration is close to the least-square solution (in accordance with Lemma 1), and the frames are almost identical after only 12 iterations. Indeed, we 

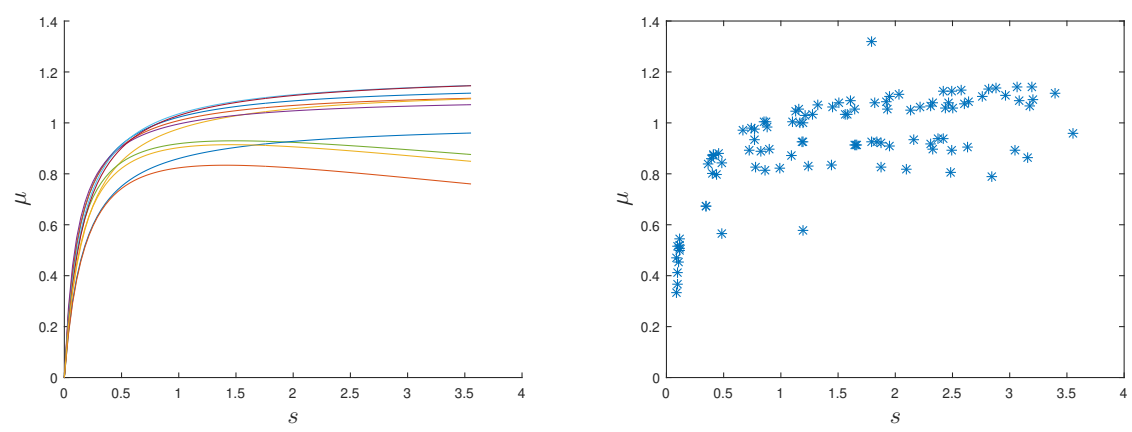

Figure 1: Growth curves randomly generated (left) and random data points $\left(s_{i}, \mu_{i}\right)$ corrupted with noise (right).
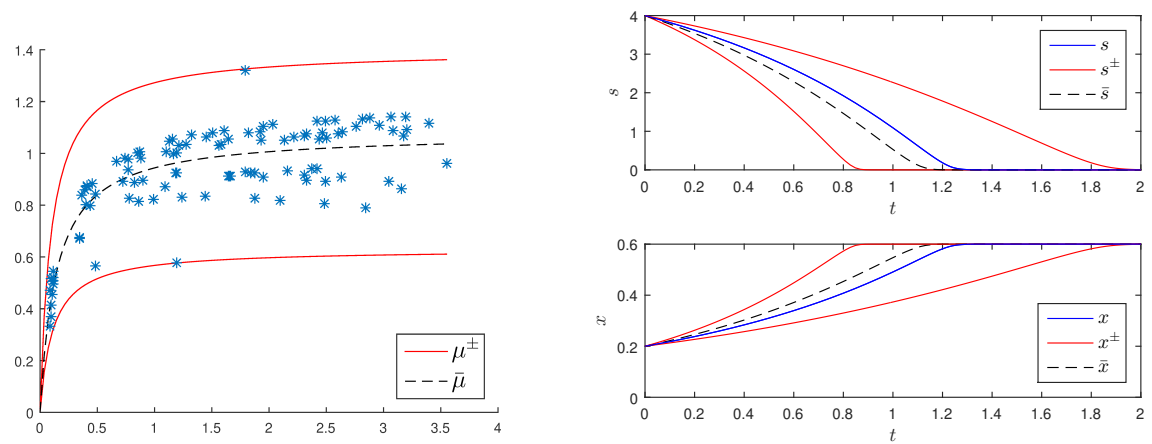

Figure 2: Wrapping of the data (left) and dynamical simulation (right).

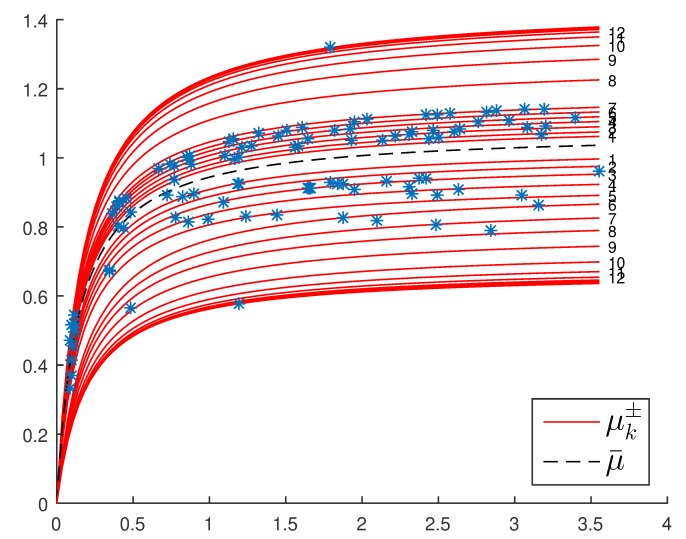

Figure 3: Convergence of the method. Functions $\mu_{k}^{-}, \mu_{k}^{+}$are obtained with pairs $\left(p_{k}^{-}, p_{k}^{+}\right)$solutions of problem $\mathcal{P}_{\gamma^{+}}$at the $k$-th iteration of Algorithm 1. 
found that it is enough to stop the algorithm when the upper estimate $\gamma^{+}$gives a solution $\left(p^{-}, p^{+}\right)$of problem $\mathcal{P}_{\gamma^{+}}$with $D\left(p^{-}, p^{+}\right)$close to $\epsilon^{2}$.

We have then simulated a trajectory of the model (28) in batch mode (i.e. for $D=0$ ) with a growth $\mu(\cdot)$ changing randomly with time, along with the solutions of the frame dynamics (31)-(32) (see Fig. 2-right). The value of the yield coefficient $r$ has been kept equal to 0.1 . One can notice that the trajectory provided by the average growth $\bar{\mu}$ (in dashed line) is not centered in the trajectories frame. This is due to the non-linearity of the dynamics. This shows the interest of the information provided by the guaranteed interval approach, differently to confidence intervals of the least-square method that are centered on the trajectory generated by the average growth.

One may want to relax the growth frame considering that some data points are potentially outliers. For this purpose, we have applied the extension of the method presented in Section 3 for different values of $j$, the number of unwrapped points. On Fig. 4, one can observe the reduction obtained on the value of $D\left(p_{j}^{\star-}, p_{j}^{\star+}\right)$ with the pair $\left(p_{j}^{\star-}, p_{j}^{\star+}\right)$ provided by Algorithm 2 for $j$ unwrapped points (remind that $D\left(p_{j}^{\star-}, p_{j}^{\star+}\right)$ is a measure of the frame width). This gives insights on the geometrical distribution of the data points. For this data set, it

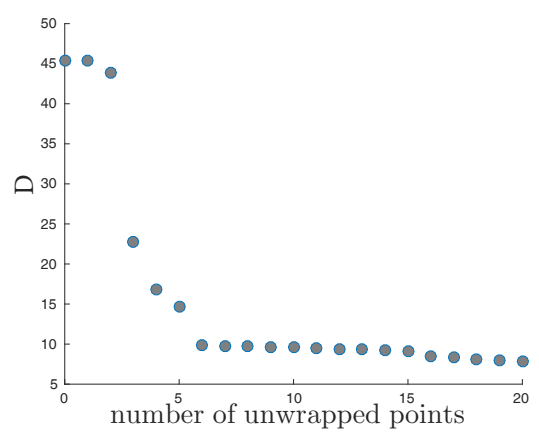

Figure 4: Sensitivity of $D\left(p_{j}^{\star-}, p_{j}^{\star+}\right)$ (where $\left(p_{j}^{\star-}, p_{j}^{\star+}\right)$ is given by Algorithm 2) with respect of the number $j$ of data points allowed to be unwrapped.

can be seen that withdrawing only three points (among one hundred) reduces significantly the frame width. Indeed, the algorithm gave the same sequence of frames as shown in Fig. 3 up to the ninth iteration, which provides a frame that excludes exactly three data points, represented in Fig. 5-left. In Fig. 5-right one can appreciate the shrinking of the trajectories frame in the transients.

Guaranteed estimations of transients are of primer importance in industrial applications. In the batch process, the asymptotic value of the biomass $x$ is known, equal to $x_{\infty}=x_{0}+r s_{0}$ according to (30), but guaranteed intervals allow to estimate the time necessary to reach a given level of production $x_{l}<x_{\infty}$.

We have also tested the method on a data set with a limited number of experimental points. On Fig. 6-left, data has been generated from three possible curves with a total of ten measurements. The wrapping obtained with all data is depicted in red, and in green when excluding one data point. On Fig. 6-right, one can see a dynamical simulation of the system, with the frames obtained with the two different wrappings. Although the green wrapping is quite close 

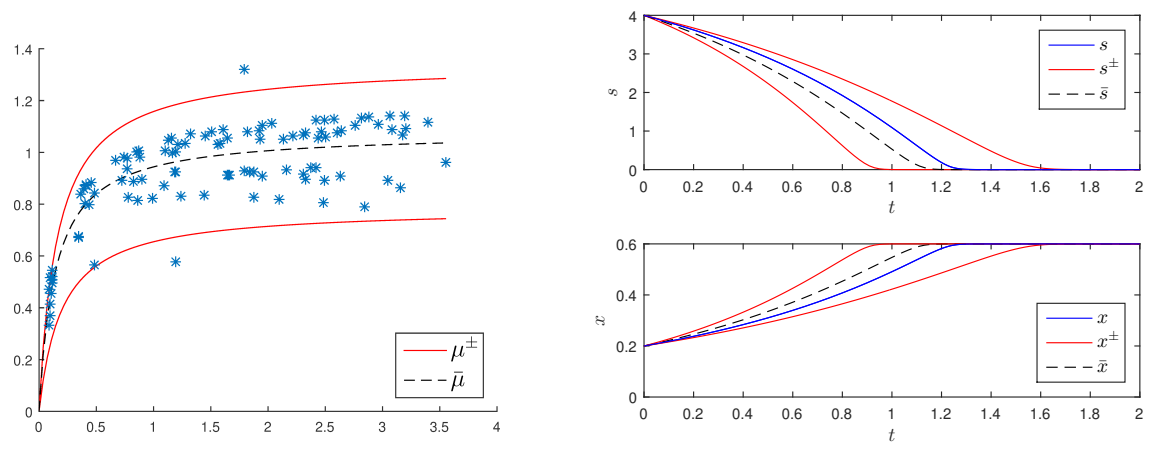

Figure 5: Data wrapping (left) and corresponding simulation (right) allowing three data points to be out of the frame.

to the red one, the true trajectory of the system does not belong to the frame generated by the green wrapping. Indeed, when there are few points, it is more likely to obtain a growth curve that lies on the boundary of the set of experimental points, and then the frame is less robust to the relaxation with unwrapped points. Notice that in this case, the least-square estimation is quite far from the true trajectory (and also far from the center of the intervals). This example shows the possible limitations of the method with small data sets: the frame obtained with all the data does always contain the true trajectory but could be quite conservative, and relaxed ones could be unsatisfactory.
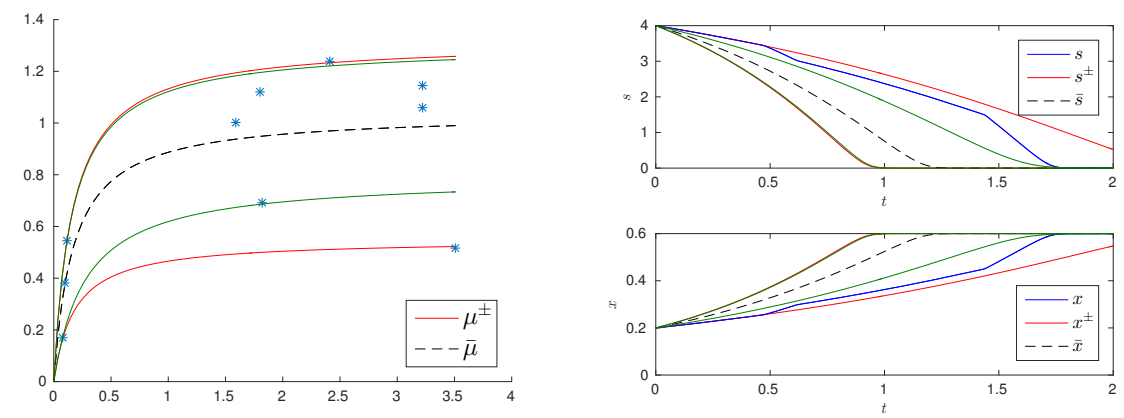

Figure 6: Data wrapping (left) and simulation (right) with only ten experimental data points (in red: with all the data; in green: with exclusion of one point.

\subsection{Example of a continuous process}

We have generated another set of random growth curves and random data (see Fig. 7).

As previously, we have wrapped all the data points in between the graphs of two functions $\mu^{-}, \mu^{+}$among the class of Monod growth functions, depicted on Fig. 8-left (the average growth determined by the least-square criterion is plotted 

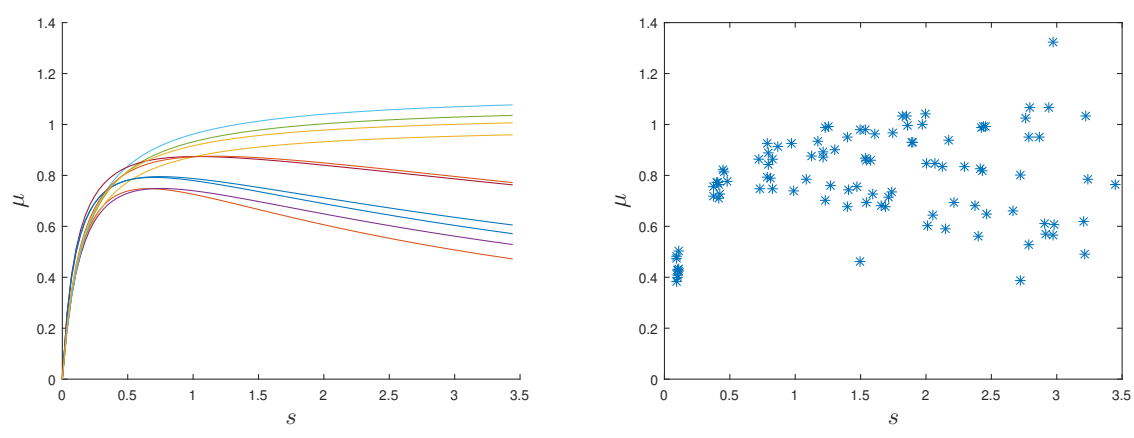

Figure 7: Growth curves randomly generated (left) and random data points $\left(s_{i}, \mu_{i}\right)$ corrupted with noise (right).

in dashed line). We have then considered the model (28) in continuous mode for the input parameters $D=0.7$ and $s_{i n}=4$ (with the same yield coefficient $r=0.1$ ). Fig. 8-right shows a trajectory for a growth $\mu(\cdot)$ changing randomly with time, lying inside the guaranteed time-varying intervals computed with the functions $\mu^{-}, \mu^{+}$.
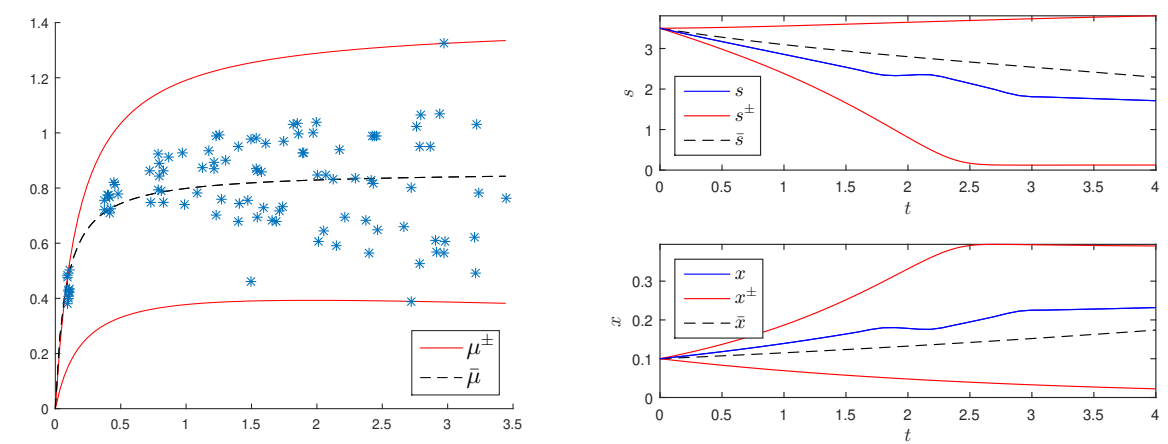

Figure 8: Wrapping with Monod growth functions (left) and associated dynamical simulation (right)

To illustrate the flexibility of the method presented in Section 3, we have also looked for lower functions $\mu^{-}$in the class of Haldane functions (29), allowing thus non-monotonic growth curves (see Fig. 9). The area criterion (26) gives an improvement of the fitting (2.66 instead of 3.01). However, this does not reduce too much the trajectories frame as this concerns only small values of $s$ (see Fig. 10-right). Also, we have applied the extension of the method (Algorithm 2) allowing some data points to be unwrapped. Fig. 11 gives insights on the data and shows how many points need to be withdrawn to obtain tighter frames. For height unwrapped data points, one can observe a significant shrinking of the growth frame (see Fig. 12-left) as well as the trajectories frame in the transients (see Fig. 12-right). Here also, one can see that the framing method is a relevant tool to obtain safe transients estimates, while the use of the least-square method is more questionable in the context of unpredictable variations. 


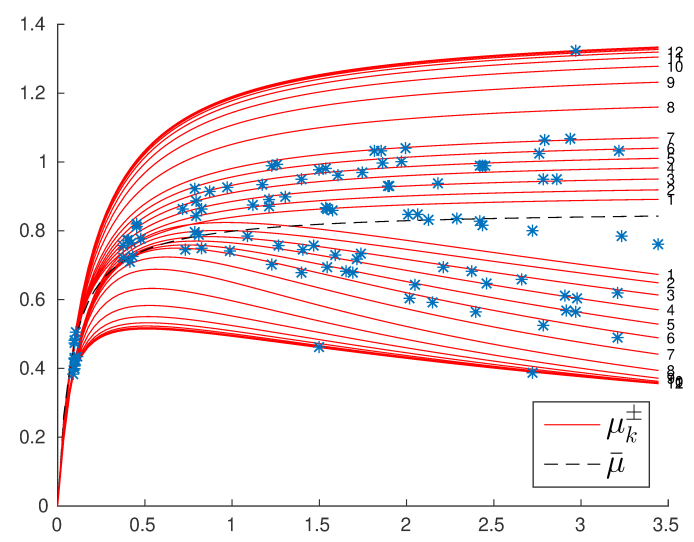

Figure 9: Convergence of the method. Functions $\mu_{k}^{-}, \mu_{k}^{+}$are obtained with pairs $\left(p_{k}^{-}, p_{k}^{+}\right)$solutions of problem $\mathcal{P}_{\gamma^{+}}$at the $k$-th iteration of Algorithm 1.
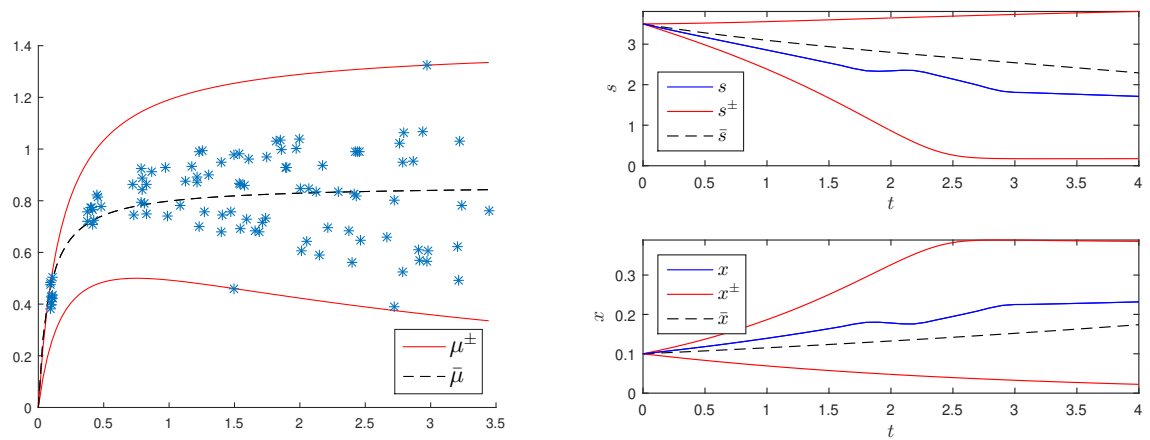

Figure 10: Wrapping with Monod and Haldane growth functions (left) and associated simulation (right)

Of course, better trajectories frames could be obtained when on-line measurements are available, using "interval observers" as recalled in the introduction. To illustrate the use of the growth framing in such a context, we have considered a situation where the substrate concentration alone is measured in the output flow rate of the reactor and the initial biomass is unknown (assuming that a rough interval could be provided). Fig. 13 shows simulations of the interval observer proposed in [45] with the same trajectory as previously simulated, with and without measurement noise. This shows the interest of having dynamic measurements, in particular, to estimate with accuracy the final biomass production. 


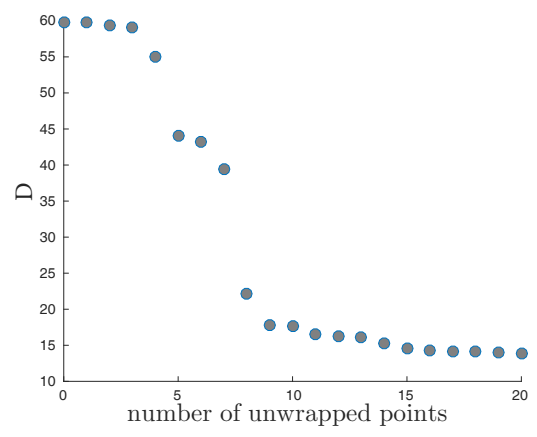

Figure 11: Sensitivity of $D\left(p_{j}^{\star-}, p_{j}^{\star+}\right)$ (where $\left(p_{j}^{\star-}, p_{j}^{\star+}\right)$ is given by Algorithm 2 ) with respect of the number $j$ of data points allowed to be unwrapped.
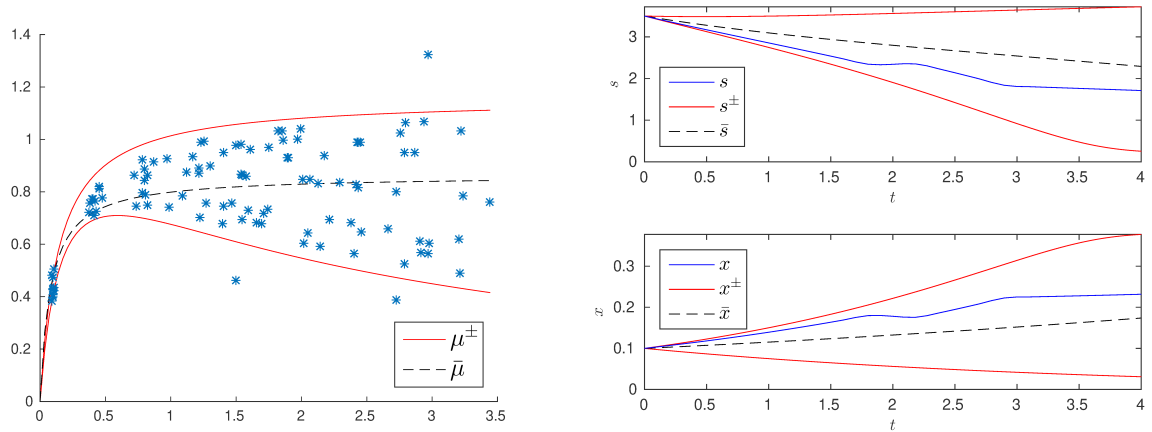

Figure 12: Data wrapping (left) and corresponding simulation (right) allowing height data points to be out of the frame.
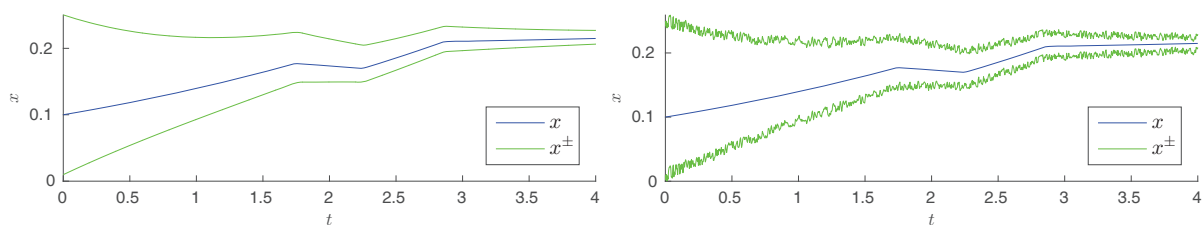

Figure 13: Bounds on the biomass concentration obtained with the observer proposed in [45] with gains $G_{1}=1, G_{2}=-10$ without measurement noise (left) and with a $10 \%$ uniform noise (right).

\section{Illustration on real data}

In this section, we apply our method on real data obtained from laboratory experiments. The experiments were conducted in batch microalgae cultures of $40 \mathrm{ml}$ working volume at fixed initial conditions of substrate $s_{0}$ and biomass $x_{0}$, under a fixed temperature of $25^{\circ} \mathrm{C}$ and under different $p H$ conditions (from 6 to 9 ). The biomass was tracked daily, either by optical density (at $650 \mathrm{~nm}$ ) or 
by fluorescence (EX 450nm, EM 680 $\mathrm{nm}$ ), and then converted into carbon unit, while the substrate TAN (Total Ammonia Nitrogen) concentrations were measured with a spectro-photometric test kit. This experimental procedure allowed to test a significant number of growth rates depending on the chosen initial condition of $s(0)=s_{i}$ (for a set of values $s_{i}$ ) while $x_{0}$ was kept constant. The growth rates have been estimated by the determination of the slopes of $t \log x(t)$ at initial time, from the measure of the variation of $x($.$) during the time pe-$ riod 0 to $48 \mathrm{~h}$. The data set comprises 88 data points obtained by aggregating the results of different series of experiments carried out on the same microalgae species (Chlorella sorokiniana) under different ranges of TAN [23], with replicates. The yield factor $r$ was determined from the available data of both substrate and biomass concentrations $\left(s_{f}, x_{f}\right)$ measured at the end of growth with a few experiments, by the expression $\frac{x_{f}-x_{0}}{s_{0}-s_{f}}$, providing a total of 37 data points.

From the data analysis, we found that uncertainties appear to be within both growth rates and yield factors, along the range of environmental conditions and tested substrate concentrations. Thus, we propose a double application of our framing method for the two data sets before exploiting the model with intervals simulations in batch culture. For the micro-algae growth rates data set, the fluctuations in measurements can be due to the different $p H$ or to measurement errors. We choose to apply our framing method using the class of Monod functions (8) for the upper and lower bounds $\mu^{-}$and $\mu^{+}$. For yields data set, uncertainties faced were common to low concentrations, which may be explained by the presence of dissolved organic nitrogen (not measured in these experiments) that can be consumed by micro-algae when the mineral nitrogen $s_{0}$ is depleted. From a quick glance at the data, we found the following class of functions to be appropriate to wrap yield data: $r(s)=-A \ln (s)+B$ (with parameters $A$ and $B$ such that $r$ is positive on the domain of considered $s$ ) and provide the upper and lower functions $r^{-}$and $r^{+}$.

To obtain guaranteed predictions of $x($.$) and s($.$) , we consider the model (28)$ with the parameter $D=0$ and with a variable yield $r=r(s)$, that is

$$
\left\{\begin{array}{l}
\dot{x}=\mu(s) x \\
\dot{s}=-\frac{\mu(s) x}{r(s)}
\end{array}\right.
$$

From equations (33), one has $\dot{x}+r(s) \dot{s}=0$. Thus, we can write $\dot{x}=-r(s) \dot{s}=$ $\frac{d}{d t} R(s(t))$, where the function $R$ is defined as follows:

$$
R(s)=\int_{0}^{s} r(\sigma) d \sigma .
$$

Note that $R$ is an increasing function as long as $r$ is positive. Therefore, the frame $r^{-}(\cdot) \leq r(\cdot) \leq r^{+}(\cdot)$ gives $R^{-}(s) \leq R(s) \leq R^{+}(s)$.

Let $z=R(s)+x$, then one has $\dot{z}=0$ and the parameter $z$ is framed by

$$
z^{-}:=R^{-}\left(s_{0}\right)+x_{0} \leq z \leq z^{+}:=R^{+}\left(s_{0}\right)+x_{0}
$$

Applying Muller's Theorem [40] on system (33), taking into consideration that the frame on the function $R(\cdot)$ gives also the inequalities

$$
z^{-}-R^{+}(s) \leq x(t) \leq z^{+}-R^{-}(s),
$$


leads to the following dynamics

$$
\left\{\begin{array}{l}
\dot{x}^{-}=\mu^{-}\left(s^{-}\right) x^{-}, x^{-}(0)=x_{0} \\
\dot{x}^{+}=\mu^{+}\left(s^{+}\right) x^{+}, x^{+}(0)=x_{0} \\
\dot{s}^{-}=-\frac{\mu^{+}\left(s^{-}\right)}{r^{-}\left(s^{-}\right)} \min \left(x^{+}, z^{+}-R^{-}\left(s^{-}\right)\right), s^{-}(0)=s_{0} \\
\dot{s}^{+}=-\frac{\mu^{-}\left(s^{+}\right)}{r^{+}\left(s^{+}\right)} \max \left(x^{-}, z^{-}-R^{+}\left(s^{+}\right)\right), s^{+}(0)=s_{0}
\end{array}\right.
$$

which ensures that the frames of $s^{-}(t) \leq s(t) \leq s^{+}(t)$ and $x^{-}(t) \leq x(t) \leq x^{+}(t)$ are fulfilled.

Figure 14 shows the results when wrapping all the data. Due to the double uncertainty on $\mu$ and $r$, the dynamical bounds are quite conservative. However, allowing the method to select $70 \%$ of the data shows a significant reduction of the frame width in Fig. 15-down, compared to Fig. 14-down where $100 \%$ of data points are wrapped. Tables 1 and 2 gather all the corresponding parameters estimates of the growth and yield functions respectively, where $\hat{\mu}$ and $\hat{r}$ were obtained using the least square method and $\mu^{-}, \mu^{+}$and $r^{-}, r^{+}$by applying our framing method. Finally, we have noticed that the dynamical data can break the simulated boundaries when the method excludes more than $50 \%$ of the data points.

\begin{tabular}{c|ccc}
$\begin{array}{c}\text { parameters } \\
\text { interval search }\end{array}$ & $\begin{array}{c}\mu_{\max }\left(d^{-1}\right) \\
{[0,5]}\end{array}$ & $\begin{array}{c}K_{s}\left(m g N . L^{-1}\right) \\
{[0,10]}\end{array}$ & wrapped data points $(\%)$ \\
\hline$\hat{\mu}$ & 0.90 & 0.34 & 100 \\
\hline$\mu^{-}$ & 0.65 & 5.55 & 100 \\
$\mu^{+}$ & 1.23 & 0.46 & 100 \\
\hline$\mu^{-}$ & 0.78 & 0.35 & 70 \\
$\mu^{+}$ & 1.11 & 0.46 & 70 \\
\hline
\end{tabular}

Table 1: Parameters estimates for the growth function.

\begin{tabular}{c|ccc}
$\begin{array}{c}\text { parameters } \\
\text { interval search }\end{array}$ & $\begin{array}{c}A \\
{[0,100]}\end{array}$ & $\begin{array}{c}B \\
{[0,200]}\end{array}$ & wrapped data points (\%) \\
\hline$\hat{r}$ & 5.14 & 26.71 & 100 \\
\hline$r^{-}$ & 2.22 & 12.21 & 100 \\
$r^{+}$ & 6.23 & 36.23 & 100 \\
\hline$r^{-}$ & 3.26 & 17.03 & 70 \\
$r^{+}$ & 6.30 & 34.29 & 70 \\
\hline
\end{tabular}

Table 2: Parameters estimates for the yield function.

\section{Conclusion and perspectives}

We have proposed a geometrical method for the construction of functional frames from experimental data points and applied it to microbial growths, which 

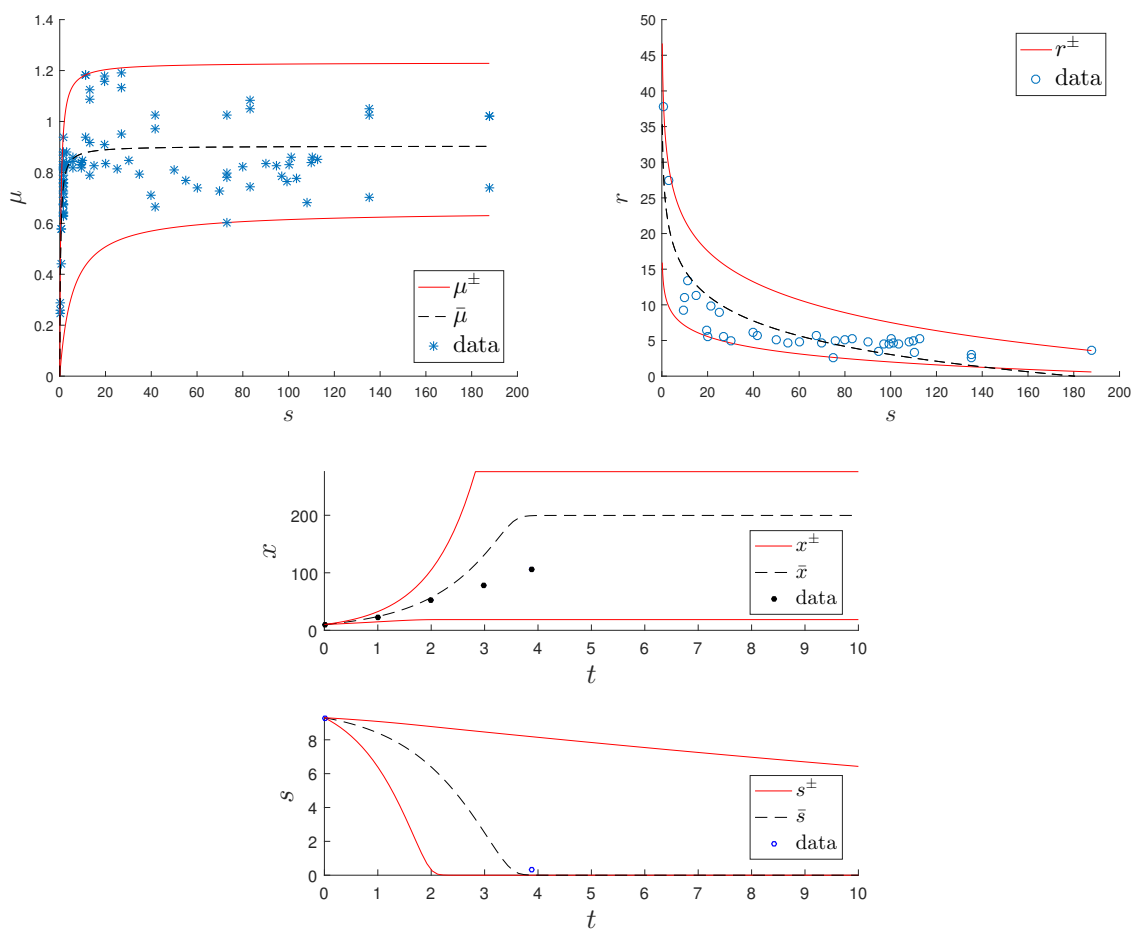

Figure 14: Wrapping 100\% of data of micro-algae growth rates (left up) and $100 \%$ yields data (right up) and associated dynamical simulation in batch culture, along with data points (down).

are often the major source of variability in bioprocesses for robust predictions. Our approach consists in computing a sequence of simple unconstrained optimization problems, allowing flexibility in the choice of upper and lower classes of functions and the number of data points that could be unwrapped. Let us underline that it does not consist in determining a set (or intervals) of parameters values in a class of functions, but rather in wrapping the data in between the graphs of two functions without requiring any particular shape of the unknown growth function (that could moreover change with time). However, the growth functions may depend on other variables, such as biomass concentration in the Contois model, or could be multi-valued, such as in the Droop model where the nutrient uptake function $\rho(\cdot)$ has to be identified concomitantly to the growth rate function $\mu(\cdot)$. Extensions of the method for these multi-valued contexts might be the matter of a future work.

\section{Acknowledgments}

This work was supported by the LabEx NUMEV incorporated into the I-Site MUSE funded by the French Research Agency (ANR), and the ADEME French Agency.This study was also supported by the PHYCOVER project, which was 

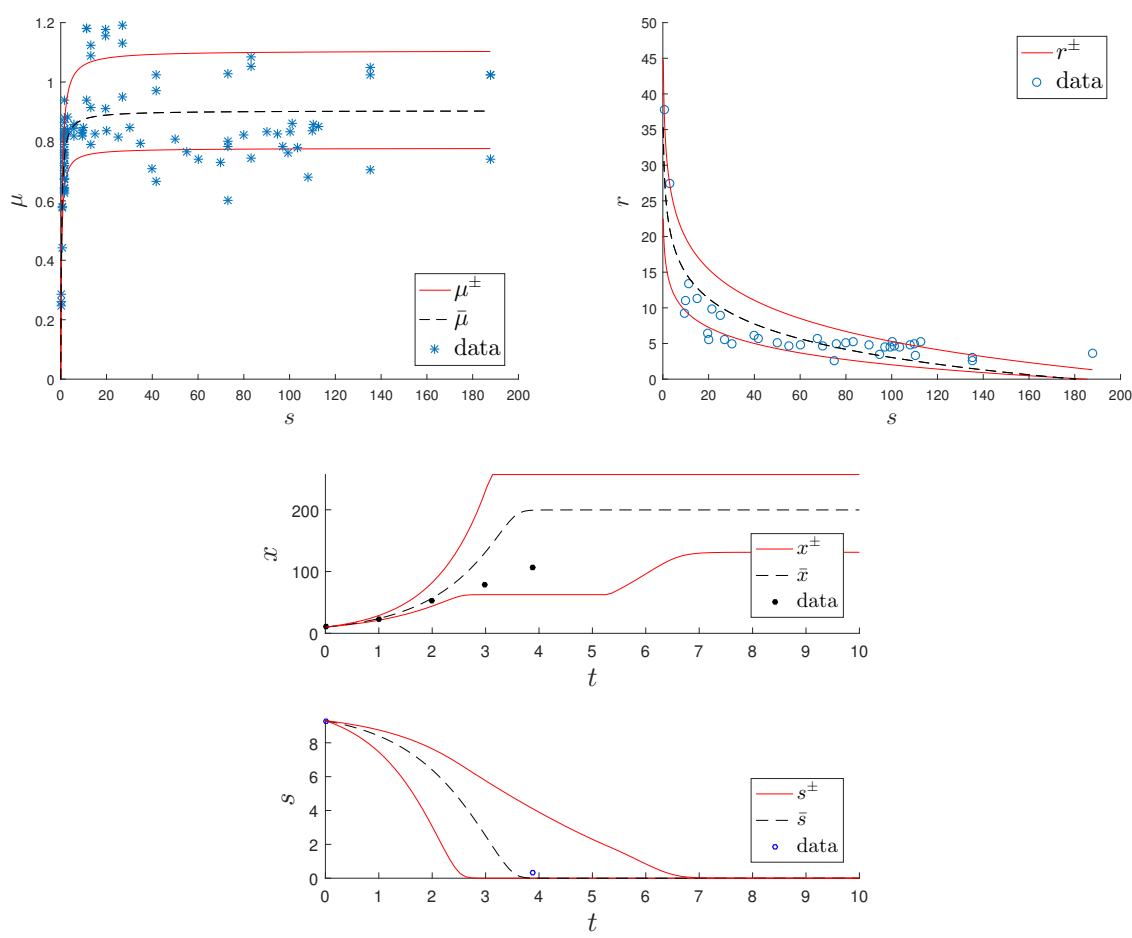

Figure 15: Wrapping $70 \%$ of data of micro-algae growth rates (left up) and 70\% yields data (right up) and associated dynamical simulation in batch culture, along with data points (down)

funded by the French National Agency for Research (ANR-14-CE04-0011).

\section{References}

[1] Alcaraz-Gonzalez, V., Harmand, J., Rapaport, A., Steyer J.P., Gonzalez-Alcarez, V. and Pelayo-Ortiz, C. Software sensors for higly uncertain WWTPs: a new approach based on intervals observers, Water Research, 36, 2515-2524, 2002.

[2] Andrews, J. A mathematical model for the continuous culture of microorganisms utilizing inhibitory substrates. Biotechnology and Bioengineering, 10, 6, 707-723, 1968.

[3] Bastin, G. And Dochain, D. On-line estimation and adaptive control of bioreactors, Elsevier, 1990.

[4] Bernard, O. ANd GouzÉ, J.-L. Closed loop observers bundle for uncertain biotechnological models. Journal of Process Control 14 (7), 765-774, 2004 . 
[5] Bernard, O., GouzÉ, J.-L. And HADJ-SAdOK, Z. Observers for the biotechnological processes with unknown kinetics. Application to wastewater treatment. In: Proceedings of the 39th IEEE Conference on Decision and Control, Sydney (Australia), 2000.

[6] Bunciu, E. Biomass Estimation for an Anaerobic Bioprocess Using Interval Observer In: Proceedings of the 3rd International on Intelligent Decision Technologies (IDT'2011) Watada J., Phillips-Wren, G., Jain, L. and Howlett, R. (Eds), Smart Innovation, Systems and Technologies, Springer, 95-102, 2011.

[7] Dochain, D. Automatic Control of Bioprocesses. (Editor) Control Systems, Robotics and Manufacturing series, ISTE-Wiley, 2008.

[8] Efimov, D., Raissi, T., Chebotarev, S., Zolghadri, A. Interval state observer for nonlinear time varying systems. Automatica, 49(1), 200-205, 2013

[9] Goffaux, G. And Vande Wouwer, A. Bioprocess State Estimation: Some Classical and Less Classical Approaches. In: Control and Observer Design for Nonlinear Finite and Infinite Dimensional Systems. Springer, 111-128, 2005.

[10] Goffaux, G., Vande Wouwer, A. And Bernard, O. Continuousdiscrete interval observers for monitoring microalgae cultures. Biotechnology progress 25 (3), 667-675, 2009.

[11] Goodwin, G. And Payne, R. Dynamic System Identification, Experiment Design and Data Analysis. Academic Press, New York, 1977.

[12] Gottu Muknula, A. And Paulen, R. Model-based design of optimal experiments for nonlinear systems in the context of guaranteed parameter estimation, Computers \& Chemical Engineering, 99, 198-213, 2017.

[13] Gouzé, J.-L., Rapaport, A. And Hadj-SAdOK, Z. Interval observers for uncertain biological systems, Ecological modelling, 133, 45-56, 2000.

[14] Gucik-Derigny, D., Raissi, T. And Zolghadri, A. A note on interval observer design for unknown input estimation. Int. J. Control, 89 (1), 25$37,2016$.

[15] Hadj-SAdok, Z. And Gouzé J.-L. Estimation of uncertain models of activated sludge processes with interval observers. Journal of Process Control, 11 (3), 299-310., 2001.

[16] Harmand, J., Lobry, C., Rapaport A. And Sari, T., The chemostat, mathematical theory of continuous culture of micro-organisms, ISTE Press, London, 2017.

[17] JAulin L. Nonlinear Bounded-Error State Estimation of Continuous-Time Systems. Automatica, 38 (6), 1079-1082., 2002.

[18] Jaulin, L., Kieffer, M., Didrit, O. And Walter, E. Applied Interval Analysis: with examples in parameter and state estimation, robust control and robotics. Springer-Verlag, 2001. 
[19] JAULIN, L. AND WALTER, E. Guaranteed nonlinear parameter estimation from bounded-error data via interval analysis, Mathematics and computers in simulation, 35, 2, 123-137, 1993.

[20] Jaulin, L., Walter E. An Didrit, O. Guaranteed robust nonlinear parameter bounding, CESA'96 IMACS Multiconference Symposium on Modelling, Analysis and Simulation, Lille (France), 2, 1156-1161, 1996.

[21] KiefFer, M. AND WALter, E. Guaranteed nonlinear state estimator for cooperative systems. Numerical Algorithms, 37, 187-198, 2004.

[22] Kieffer, M., Walter, E. And Simeonov, I. Guaranteed non-linear parameter estimation for continuous-time dynamical models. In: Proceedings 14th IFAC Symposium on System Identification, Newcastle (Australia), 843-848, 2006.

[23] Krichen, E., Rapaport, A., Le Floc'H, E. And Fouilland, E. Demonstration of facilitation between microalgae to face ammonia toxicity. Research report hal.archives-ouvertes.fr/hal-02162562/, 2019.

[24] De Leenheer, P., Angeli D. And Sontag, E., A Tutorial on Monotone Systems - With an Application to Chemical Reaction Networks. In Proc. 16th Int. Symp. Macd thematical Theory of Networks and Systems (MTNS), Leuven (Belgium), 2004.

[25] Lohner, R. Computation of guaranteed enclosures for the solutions of ordinary initial and boundary value-problem. In: Cash, J. and Gladwell I. (Eds.) Computational Ordinary Differential Equations, Clarendon Press, Oxford, 425-435, 1992.

[26] Mairet, F., Moisan, M. And Bernard, O. Interval observer-based estimator of specific growth rate in bioreactors. Journal Européen des Systèmes Automatisés (JESA), Lavoisier, 44 (4-5), 493-507, 2010.

[27] Marvel, S. And Williams, C. Set membership experimental design for biological systems, BMC systems biology, 6, 1, 21, 2012.

[28] Mazenc, F. AND BeRnARD, O. ISS interval observers for nonlinear systems transformed into triangular systems. Int. J. Robust Nonlinear Control, 24 (7), 1241-1261, 2014

[29] Meslem, N., Ramdani, N. And Candau, Y. Interval Observers for Uncertain Nonlinear Systems. Application to Bioreactors. In: Proceedings of the 17th World Congress, The International Federation of Automatic Control Seoul (Korea) July 6-11, 9667-9672, 2008.

[30] Meslem, N., Ramdani, N. And Candau, Y. Approximation garantie de l'espace d'état atteignable des systémes dynamiques continus incertains. Encadrement par intervalle de flot d'état. Journal Euroéen des Systémes Automatisés (JESA), 43, 589-614, 2009.

[31] Meslem, N., Ramdani, N. and Candau, Y. Using hybrid automata for set-membership state estimation with uncertain nonlinear continuous-time systems. Journal of Process Control, 20, 481-489., 2010. 
[32] Milanese, M. Properties of least squares estimates in set membership identification. Automatica, 31, 2, 327-332, 1995.

[33] MoIsAn, M. And Bernard, O. Interval observers for non monotone systems. Application to bioprocess models. IFAC Proceedings 38 (1), 43-48, 2005.

[34] Moisan, M. And Bernard, O. Near optimal interval observers bundle for uncertain bioreactors. Automatica, 45 (1):291-295, 2009

[35] Moisan, M. And Bernard, O. Robust interval observers for global Lipschitz uncertain chaotic systems. Systems \& Control Letters 59 (11), 687 $694,2010$.

[36] Monod, J. La technique de culture continue, théorie et applications, Ann. Inst. Pasteur, 79, 390-410,1950.

[37] Moore, R. Interval analysis. Prentice-Hall, Englewood Cliffs, 1966.

[38] Moore, R. Parameter sets for bounded-error data. Mathematics and Computers in Simulation, 34, 2, 113-119, 1992.

[39] Moser, H. The Dynamics of Bacterial Populations Maintained in the Chemostat, Carnegie Institution of Washington Publication, 1958.

[40] Muller, M. Uber das Fundamentaltheorem in der Theorie der gewohnlichen Differential-gleichungen. Math. Z., 619-645, 1927.

[41] Perić, N., Paulen, R., Villanueva, M. And Chachuat, B. Setmembership nonlinear regression approach to parameter estimation. Journal of Process Control, 70, 80-95, 2018.

[42] Raissi, T., Efimov, D. And Zolghadri, A. Interval state estimation for a class of nonlinear systems. IEEE Transactions on Automatic Control, 57 (1), pp. 260-265, 2012.

[43] Raissi, T., Ramdani, N. And Candau, Y. Set membership state and parameter estimation for systems described by nonlinear differential equations. Automatica, 40 (10), 1771-1777., 2004.

[44] Raissi, T., Ramdani, N. And Candau, Y. Bounded-error moving horizon state estimator for non-linear continuous-time systems : application to a bioprocess system. Journal of Process Control, 15 (5), 537-545, 2005.

[45] RAPAPORT, A AND DOCHAIN, D. Interval observers for biochemical processes with uncertain kinetics and inputs. Mathematical biosciences, 193 (2):235-253, 2005.

[46] Rapaport, A. And Harmand, J. Robust regulation of a class of partially observed nonlinear continuous bioreactors. Journal of Process Control, 12, 291-302, 2002.

[47] Rapaport, A. And Gouzé, J.L. Parallelotopic and practical observers for nonlinear uncertain systems. Int. Journal. Control, 76 (3), 237-251, 2003. 
[48] Ratkowsky, D. Model fitting and uncertainty, In: Modeling Microbial Responses in Food. McKellar, R., Lu, X. (Eds.), CRC Press, Boca Raton, 152-196, 2004.

[49] Rauh, A. And Auer, E. Modeling, Design, and Simulation of Systems with Uncertainties. Mathematical Engineering series, Springer 2011.

[50] Robinson, J. Determining microbial kinetic parameters using non-linear regression analysis. Adv. Microb. Ecol., 8, 61-114 (1985).

[51] Ruszczyński, A. Nonlinear Optimization. Princeton University Press, 2006.

[52] Singer, A. And BARton, P. Bounding the solutions of parameter dependent nonlinear ordinary differential equations. SIAM J. Sci. Comput., 27 (6), 2167-2182, 2006.

[53] SchitTkowski, K. Numerical Data Fitting in Dynamical Systems, A Practical Introduction with Applications and Software, Springer, 2002.

[54] Smith, H. Monotone Dynamical Systems. American Mathematical Society, Providence, 1995.

[55] Thabet, R., Raissi, T., Combastel, C., Efimov, D. And Zolghadri, A. An effective method to interval observer design for time-varying systems. Automatica, 50 (10), 2677-2684, 2014.

[56] Walter, W. Differential inequalities and maximum principles: theory, new methods and applications. Nonlinear analysis, Theory, Methods and Applications, 30(8), 4695-4711, 1997.

[57] Walter, E. AND Piet-LAhAnier, H. Estimation of parameter bounds from bounded-error data: a survey. Mathematics and Computers in Simulation, 32, 5-6, 449-468, 1990.

[58] Zheng, G., Efimov, D. And Perruquetti, W. Design of interval observer for a class of uncertain unobservable nonlinear systems. Automatica, 63, 167-174, 2016. 